\title{
TITLE:
}

\section{Energy density concept: A stress tensor approach}

\author{
$\operatorname{AUTHOR}(\mathrm{S}):$ \\ Tachibana, Akitomo
}

\section{CITATION:}

Tachibana, Akitomo. Energy density concept: A stress tensor approach. Journal of Molecular Structure: THEOCHEM 2010, 943(1-3): 138-151

\section{ISSUE DATE:}

2010-03-15

URL:

http://hdl.handle.net/2433/124569

\section{RIGHT:}

Copyright (C) 2009 Elsevier B.V.; この論文は出版社版でありません。引 用の際には出版社版をご確認ご利用ください。; This is not the published version. Please cite only the published version. 


\title{
Energy density concept: a stress tensor approach
}

\author{
Akitomo Tachibana \\ Department of Micro Engineering, Kyoto University, Kyoto 606-8501, JAPAN \\ E-mail : akitomo@scl.kyoto-u.ac.jp
}

(received: 21 August 2009)

\begin{abstract}
Conceptual insights from the density functional theory have been enormously powerful in the fields of physics, chemistry and biology. A natural outcome is the concept of "energy density" as has been developed recently: drop region, spindle structure, interaction energy density. Under external source of electromagnetic fields, charged particles can be accelerated by Lorentz force. Dissipative force can make the state of the charged particles stationary. In quantum mechanics, the energy eigenstate is another rule of the stationary state. Tension density of quantum field theory has been formulated in such a way that it can compensate the Lorentz force density at any point of space-time. This formulation can give mechanical description of local equilibrium leading to the quantum mechanical stationary state. The tension density is given by the divergence of stress tensor density. Electronic spin can be accelerated by torque density derived from the stress tensor density. The spin torque density can be compensated by a force density, called zeta force density, which is the intrinsic mechanism describing the stationary state of the spinning motion of electron.
\end{abstract}

\section{Key Words}

energy density; stress tensor; spin torque 


\section{Introduction}

The idea of the density functional theory (DFT) by Hohenberg-Kohn [1] is that with the external potential $v(\vec{r})$ given, the electronic ground state energy is a unique functional of the electron number density $n(\vec{r})$ :

$$
\begin{aligned}
& E[n(\vec{r})]=F[n(\vec{r})]+\int d^{3} \vec{r} v(\vec{r}) n(\vec{r}), \\
& \int d^{3} \vec{r} n(\vec{r})=N,
\end{aligned}
$$

where $F[n(\vec{r})]$ is the universal functional of $n(\vec{r})$ integrated to give the total electron number $N$. The external potential $v(\vec{r})$ is the clamped-nuclei one-electron electrostatic potential. The idea was extended to grand canonical ensemble by Mermin [2].

Conceptual insights from the DFT have been enormously powerful in the fields of physics, chemistry, and biology [3]. A natural outcome is the realization of $\Psi[n(\vec{r})]$ and $\rho[n(\vec{r})]$, which means that the wave function and the density matrix are given as the functionals of $n(\vec{r})$.

Based on this realization, the concept of "energy density" has been developed recently using "stress tensor" machineries [4-12]. The energy density concept has been essential in the field theory with the Hamiltonian density operator as shown in Fig. 1 and the stress tensors are used ubiquitously for description of internal forces of matter. They have been originally formulated by Pauli [13] in quantum mechanical context with the differential force law showing that it can be derived from the divergence relations applied to the energy-momentum tensor under general situations in the presence of electromagnetic fields, while the basic idea dates back to Schrödinger [14], and recently developed by Bader [15] in his Atoms-in-Molecules (AIM) theory and Epstein [16] in his hypervirial theory. 


\section{Field theory of energy density concept}

\begin{tabular}{|c|c|c|}
\hline \multicolumn{2}{|c|}{$\begin{array}{l}\text { Hamiltonian density } \\
\text { operator } \\
\qquad \hat{H}(\vec{r})\end{array}$} & $\begin{array}{l}\text { Hamiltonian } \\
\text { operator } \\
\qquad \hat{H}=\int d^{3} \vec{r} \hat{H}(\vec{r})\end{array}$ \\
\hline $\begin{array}{l}\text { expectation } \| \\
\text { value }<>\end{array}$ & \multicolumn{2}{|c|}{ integral $\int d^{3} \vec{r}$} \\
\hline $\begin{array}{l}\text { Energy d } \\
\qquad n_{H}(\vec{r})=\end{array}$ & & $\begin{array}{l}\text { Energy } \\
\qquad E=\int d^{3} \vec{r} n_{H}(\vec{r})=\langle\hat{H}\rangle\end{array}$ \\
\hline
\end{tabular}

Fig. 1. Energy density concept in the field theory.

According to the Hohenberg-Kohn theorem [1], the nonrelativistic ground state wave function is a unique functional of the electron density, so is the "stress tensor" in the field theory, and moreover, the energy density, Lorentz force density, spin torque density, and so on. Specifically for the argumentation of the regional charge transfer processes of chemical reaction coordinates, another basic strategy is the use of "Apparatus" operators [17]; then, with invoking the Mermin entropy principle for the Onsager local equilibrium hypothesis, the regional density functional theory has been developed [18] and successfully applied to the electron flow of chemical reactions [19,10-12].

Thus, we have developed the energy density concept useful in chemical applications of the DFT. The performance of the stress tensor has been proved powerful therein. The basic area of the field theory is deep in basic science even though regrettably the universal functional is not known yet. So that our approach is formal. However, since the concept is apparently natural and extremely appearing, our approach should enhance our motivation to seek for the novel breakthrough in the DFT.

The logic of the field theory is fundamental, and is briefly reviewed here as follows.

Throughout in this paper, we use the Gaussian system of units. The space-time coordinates are denoted as

$$
x=\left(x^{\mu}\right)=\left(x^{0}, x^{k}\right)=\left(x^{0}, x^{1}, x^{2}, x^{3}\right)=(c t, x, y, z)=(c t, \vec{r}),
$$

where $c$ denotes the speed of light and the Greek letter runs fron 0 to 3 and the Latin from 1 to 3. 
The contravariant vector $a^{\mu}$ is transformed to the covariant vector $a_{\mu}$ through

$$
a_{\mu}=g_{\mu v} a^{v}
$$

where the Einstein summation convention for duplicate indices is used with the metric tensor

$$
g_{\mu \nu}=\left(\begin{array}{cccc}
1 & 0 & 0 & 0 \\
0 & -1 & 0 & 0 \\
0 & 0 & -1 & 0 \\
0 & 0 & 0 & -1
\end{array}\right)=g^{\mu \nu}
$$

The divergence of tensor density $T^{\cdots k \ell}(x)$ is defined in this paper by the rule

$$
(\operatorname{div} T)^{\cdots \cdot k}(x)=\partial_{\ell} T^{\cdots k \ell}(x) .
$$

The Lagrangian density operator is composed of the field operators of gauge potentials and matter particles carrying charges and spins. Not only the electronic spin but also the nuclear spin are described by standard model of elementary particles. The nuclear spin is interacting with electron as demonstrated by NMR chemical shift or the Knight shift [20]. Positron emission tomography has been widely known in chemistry community and similar applications of positron-electron annihilation in semiconductor industries [21].

The matter particles are spin $1 / 2$ chiral Fermions in the standard model [22]. The matter particles are bound by gauge bosons obeying gauge principle. Using the standard model, the gauge fields of QCD (quantum chromodynamics) is reduced from grand unified theory (GUT) as $S U(3)_{\mathrm{c}} \times S U(2)_{\mathrm{w}} \times U(1)_{\mathrm{y}} \rightarrow S U(3)_{\mathrm{c}} \times U(1)_{\mathrm{QED}}$, where the Higgs field breaks the Weinberg-Salam electroweak gauge group $S U(2)_{\mathrm{w}} \times U(1)_{\mathrm{y}}$ down to $U(1)_{\mathrm{QED}}$, but the color and charge symmetries remain intact. Quarks are bound by gluons $G_{\mu}^{\ell}$ while electron acquires its charge and mass through the Higgs mechanism with the Yukawa coupling, when massless photon $A_{\mu} \quad$ as well as the massive bosons $Z_{\mu}{ }^{0}$ and $W_{\mu}{ }^{ \pm}$are emerging using the Glashow-Weinberg-Salam theory of spontaneously broken gauge symmetry as shown in Appendix A.

For electrons, the action through medium has been formulated in QED (quantum electrodynamics) using the gauge-invariant Lagrangian density operator $\hat{L}(x)$, 


$$
\hat{L}(x)=-\frac{1}{16 \pi} \hat{F}_{\mu \nu}(x) \hat{F}^{\mu v}(x)+\hat{L}_{e}\left(\left\{\hat{\psi}, \hat{D}_{e \mu} \hat{\psi}\right\} ; x\right)
$$

where $\hat{F}_{\mu \nu}(x)$ is the photon field density operator using Abelian transversal Coulomb gauge potentials $\hat{A}_{\mu}(x)$,

$$
\begin{aligned}
& \hat{F}_{\mu v}(x)=\partial_{\mu} \hat{A}_{v}(x)-\partial_{v} \hat{A}_{\mu}(x) \\
& =\left(\begin{array}{cccc}
0 & \hat{E}_{x}(x) & \hat{E}_{y}(x) & \hat{E}_{z}(x) \\
-\hat{E}_{x}(x) & 0 & -\hat{B}_{z}(x) & \hat{B}_{y}(x) \\
-\hat{E}_{y}(x) & \hat{B}_{z}(x) & 0 & -\hat{B}_{x}(x) \\
-\hat{E}_{z}(x) & -\hat{B}_{y}(x) & \hat{B}_{x}(x) & 0
\end{array}\right), \quad \partial_{k} \hat{A}^{k}(x)=0,
\end{aligned}
$$

and where $\hat{L}_{e}\left(\left\{\hat{\psi}, \hat{D}_{e \mu} \hat{\psi}\right\} ; x\right)$ is the Lagrangian density operator of electron,

$$
\hat{L}_{e}\left(\left\{\hat{\psi}, \hat{D}_{e \mu} \hat{\psi}\right\} ; x\right)=c \hat{\bar{\psi}}(x)\left(i \hbar \gamma^{\mu} \hat{D}_{e \mu}(x)-m_{e} c\right) \hat{\psi}(x) .
$$

with $\hat{\bar{\psi}}(x)=\hat{\psi}^{\dagger}(x) \gamma^{0}$ being the Dirac conjugate to $\hat{\psi}(x)$. The $\gamma^{\mu}$ denotes the Dirac matrices

$$
\gamma^{0}=\left(\begin{array}{ll}
0 & 1 \\
1 & 0
\end{array}\right), \quad \vec{\gamma}=\left(\begin{array}{cc}
0 & -\vec{\sigma} \\
\vec{\sigma} & 0
\end{array}\right),
$$

in the chiral representation with the Pauli spin matrices

$$
\sigma_{x}=\left(\begin{array}{ll}
0 & 1 \\
1 & 0
\end{array}\right), \sigma_{y}=\left(\begin{array}{cc}
0 & -i \\
i & 0
\end{array}\right), \sigma_{z}=\left(\begin{array}{cc}
1 & 0 \\
0 & -1
\end{array}\right),
$$

and $\hat{D}_{e \mu}(x)$ is the covariant derivative for electron,

$$
\hat{D}_{e \mu}(x)=\partial_{\mu}+i \frac{Z_{e} e}{\hbar c} \hat{A}_{\mu}(x), \quad Z_{e}=-1,
$$

with $m_{e}$ and $Z_{e}$ being the mass and charge number of electron, respectively.

QED has the Poincare and gauge symmetries. The gauge symmetry of the field theory is realized by the Becci-Rouet-Stora-Tyutin (BRST) symmetry of the Lagrangian:

$$
\partial_{\theta} \int d^{4} x \frac{1}{c} \hat{L}(x)=0,
$$

where $\partial_{\theta}$ denotes the BRST operator. It follows that the physical content of the gauge theory is consistent with the cohomology of the BRST operator. 
QED allows the clamped-nuclei Hamiltonian, where the atomic nuclei are clamped in space and are treated as external static source of force for electrons. But in chemical reaction systems, the rearrangement of atomic configuration is of primary interest, and hence the dynamical treatments of atomic nuclei have been formulated by the Rigged QED theory [4], which is an Abelian gauge theory, using the gauge-invariant Lagrangian density operator:

$$
\begin{aligned}
& \hat{L}(x)=-\frac{1}{16 \pi} \hat{F}_{\mu \nu}(x) \hat{F}^{\mu v}(x) \\
& +\hat{L}_{e}\left(\left\{\hat{\psi}, \hat{D}_{e \mu} \hat{\psi}\right\} ; x\right)+\sum_{a} \hat{L}_{a}\left(\left\{\hat{\chi}_{a}, \hat{D}_{a 0} \hat{\chi}_{a}, \hat{D}_{a k}^{2} \hat{\chi}_{a}\right\} ; x\right)
\end{aligned}
$$

$\hat{L}_{a}\left(\left\{\hat{\chi}_{a}, \hat{D}_{a 0} \hat{\chi}_{a}, \hat{D}_{a k}^{2} \hat{\chi}_{a}\right\} ; x\right)$ is the Lagrangian density operator of $a$ 'th atomic nucleus:

$$
\hat{L}_{a}\left(\left\{\hat{\chi}_{a}, \hat{D}_{a 0} \hat{\chi}_{a}, \hat{\vec{D}}_{a}^{2} \hat{\chi}_{a}\right\} ; x\right)=\hat{\chi}_{a}^{\dagger}(x)\left(i \hbar c \hat{D}_{a 0}(x)+\frac{\hbar^{2}}{2 m_{a}} \hat{\vec{D}}_{a}^{2}(x)\right) \hat{\chi}_{a}(x),
$$

where $\hat{D}_{a \mu}(x)$ is the covariant derivative of $a$ 'th atomic nucleus,

$$
\hat{D}_{a \mu}(x)=\partial_{\mu}+i \frac{Z_{a} e}{\hbar c} \hat{A}_{\mu}(x),
$$

with $m_{a}$ and $Z_{a}$ being the mass and charge number of the $a$ 'th atomic nucleus, respectively. The canonical quantization rule of the Schrödinger field is anticommutation relationship for Fermions and commutation relationship for Bosons.

The Rigged QED theory is gauge invariant and preserves translational and rotational symmetry but violates the Poincare symmetry. This is because the presence of the Schrödinger fields violates Lorentz invariance of the Lagrangian density. If we neglect the Schrödinger fields, then we recover the conventional QED with the Poincare symmetry as well as the gauge symmetry.

In the present paper, we demonstrate the energy density, force density, and spin torque density concepts in Abelian and non-Abelian gauge theories (see Appendix A). In classical mechanics, the Lorentz force is the derivative of the kinetic momentum with respect to time. In the quantum field theories, the derivative of the kinetic momentum density operator with respect to time gives the Lorentz force density operator plus the tension density operator: see Eqs. (2.32) and (2.38) in the Abelian gauge field theory and Eq. (A.23) in the non-Abelian gauge field theory. The tension density operator is given by the divergence of the stress tensor density operator: see Eqs. (2.34) and (2.39) in the Abelian gauge field theory and Eq. (A.25) in the non-Abelian gauge field theory. The derivative of the Fermion spin angular momentum density operator with respect to time gives the spin torque density operator and a force density operator, called the zeta force density operator: see Eq. (4.4) in the Abelian gauge field theory and Eq. (A.31) in the non-Abelian gauge field theory. The torque 
density operator is originated from the stress tensor density operator: see Eq. (4.5) in the Abelian gauge field theory and Eq. (A.32) in the non-Abelian gauge field theory. The zeta force density operator is originated from the gradient of the zeroth component of the chiral current density operator: see Eq. (4.7) in the Abelian gauge field theory and Eq. (A.34) in the non-Abelian gauge field theory. Phenomenological medium effects are treated in Section 5.

\section{Lorentz force density}

\subsection{General settings}

In the Rigged QED theory, the charge density conservation laws are the continuity equation

$$
\frac{\partial}{\partial t} \hat{\rho}_{e}(x)+\operatorname{div} \hat{\vec{j}}(x)=0
$$

for electron, and

$$
\frac{\partial}{\partial t} \hat{\rho}_{a}(x)+\operatorname{div} \hat{\vec{j}}(x)=0
$$

for $a$ 'th atomic nucleus. As a whole,

$$
\frac{\partial}{\partial t} \hat{\rho}(x)+\operatorname{div} \hat{\vec{j}}(x)=0,
$$

Where $\alpha=e$ stands for electron and $\alpha=a$ stands for $a$ 'th atomic nucleus; $\hat{\rho}(x)$ is the charge density operator and $\hat{\vec{j}}(x)$ is the charge current density operator. The $\hat{\rho}(x)$ is decomposed into

$$
\begin{aligned}
& \hat{\rho}(x)=\hat{\rho}_{e}(x)+\sum_{a} \hat{\rho}_{a}(x)=\sum_{\alpha} \hat{\rho}_{\alpha}(x), \\
& \hat{\rho}_{\alpha}(x)=Z_{\alpha} e \hat{N}_{\alpha}(x),
\end{aligned}
$$

where $\hat{\rho}_{e}(x)$ is the electronic charge density operator and $\hat{\rho}_{a}(x)$ is the charge density operator of $a$ 'th atomic nucleus, and where $\hat{N}_{e}(x)$ and $\hat{N}_{a}(x)$ is the position probability density operator of electron and $a$ 'th atomic nucleus, respectively:

$$
\begin{aligned}
& \hat{N}_{e}(x)=\hat{\bar{\psi}}(x) \gamma^{0} \hat{\psi}(x), \\
& \hat{N}_{a}(x)=\hat{\chi}_{a}^{\dagger}(x) \hat{\chi}_{a}(x) .
\end{aligned}
$$


The $\hat{\vec{j}}(x)$ is decomposed into

$$
\begin{aligned}
& \hat{\vec{j}}(x)=\hat{\vec{j}}_{e}(x)+\sum_{a} \hat{\vec{j}}_{a}(x)=\sum_{\alpha} \hat{\vec{j}}_{\alpha}(x), \\
& \hat{\vec{j}}_{\alpha}(x)=Z_{\alpha} e \hat{\vec{v}}_{\alpha}(x),
\end{aligned}
$$

where $\hat{\vec{j}}_{e}(x)$ is the electronic charge current density operator and $\hat{\vec{j}}_{a}(x)$ is the charge current density operator of $a$ 'th atomic nucleus, and $\hat{\vec{v}}_{\alpha}(x)$ denotes the velocity density operator:

$$
\begin{aligned}
& \hat{\vec{v}}_{e}(x)=c \hat{\bar{\psi}}(x) \vec{\gamma} \hat{\psi}(x), \\
& \hat{\vec{v}}_{a}(x)=\frac{1}{2 m_{a}}\left(i \hbar \hat{\chi}_{a}^{\dagger}(x) \hat{\vec{D}}_{a}(x) \hat{\chi}_{a}(x)-i \hbar\left(\hat{\vec{D}}_{a}(x) \hat{\chi}_{a}(x)\right)^{\dagger} \cdot \hat{\chi}_{a}(x)\right) .
\end{aligned}
$$

By Gordon decomposition, we have

$$
\begin{aligned}
& \hat{\vec{v}}_{e}(x)=\frac{1}{2 m_{e}}\left(i \hbar \hat{\bar{\psi}}(x) \hat{\vec{D}}_{e}(x) \hat{\psi}(x)-i \hbar\left(\hat{\vec{D}}_{e}(x) \hat{\psi}(x)\right)^{\dagger} \gamma^{0} \cdot \hat{\psi}(x)\right) . \\
& +\frac{\hbar}{2 m_{e}} \operatorname{rot}(\hat{\bar{\psi}}(x) \vec{\sigma} \hat{\psi}(x))-\frac{i \hbar}{2 m_{e}} \frac{\partial}{\partial t}\left(\hat{\bar{\psi}}(x) \gamma^{0} \vec{\gamma} \hat{\psi}(x)\right)
\end{aligned}
$$

The $\hat{\vec{v}}_{a}(\vec{r})$ may also be written as the flux density operator $\hat{\vec{S}}_{a}(\vec{r})$ as follows:

$$
\hat{\vec{V}}_{a}(\vec{r})=\hat{\vec{S}}_{a}(\vec{r})
$$

\subsection{Equations of motion of fields}

The equations of motion of fields is obtained using standard variational principle.

First, the Maxwell equations of motion are found for the electromagnetic fields:

$$
\begin{aligned}
& \hat{\vec{E}}(x)=-\operatorname{grad} \hat{A}_{0}(x)-\frac{1}{c} \frac{\partial \hat{\vec{A}}(x)}{\partial t}, \operatorname{div} \hat{\vec{A}}(x)=0, \\
& \hat{\vec{B}}(x)=\operatorname{rot} \hat{\vec{A}}(x)
\end{aligned}
$$

and

$$
\operatorname{rot} \hat{\vec{E}}(x)+\frac{1}{c} \frac{\partial \hat{\vec{B}}(x)}{\partial t}=0
$$




$$
\begin{aligned}
& \operatorname{div} \hat{\vec{B}}(x)=0, \\
& \operatorname{div} \hat{\vec{E}}(x)=4 \pi \hat{\rho}(x), \\
& \operatorname{rot} \hat{\vec{B}}(x)-\frac{1}{c} \frac{\partial \hat{\vec{E}}(x)}{\partial t}=\frac{4 \pi}{c} \hat{\vec{j}}(x) .
\end{aligned}
$$

Second, the Dirac spinor field satisfies

$$
\begin{aligned}
& i \hbar \gamma^{\mu} \hat{D}_{e \mu}(x) \hat{\psi}(x)=m_{e} c \hat{\psi}(x) \\
& -i \hbar\left(\hat{D}_{e \mu}(x) \hat{\psi}(x)\right)^{\dagger} \gamma^{0} \gamma^{\mu}=m_{e} c \hat{\bar{\psi}}(x) .
\end{aligned}
$$

Third, the Schrödinger field satisfies

$$
\begin{aligned}
& i \hbar \frac{\partial}{\partial t} \hat{\chi}_{a}(x)=-\frac{\hbar^{2}}{2 m_{a}} \hat{\vec{D}}_{a}^{2}(x) \hat{\chi}_{a}(x)+Z_{a} e \hat{A}_{0}(x) \hat{\chi}_{a}(x), \\
& -i \hbar \frac{\partial}{\partial t} \hat{\chi}_{a}^{\dagger}(x)=-\frac{\hbar^{2}}{2 m_{a}}\left(\hat{\vec{D}}_{a}^{2}(x) \hat{\chi}_{a}(x)\right)^{\dagger}+Z_{a} e\left(\hat{A}_{0}(x) \hat{\chi}_{a}(x)\right)^{\dagger} .
\end{aligned}
$$

\subsection{Equations of motion of momentums}

The momentum of the electromagnetic field is the Poynting vector density operator $\hat{\vec{G}}(x)$ defined as

$$
\hat{\vec{G}}(x)=\frac{1}{4 \pi c} \hat{\vec{E}}(x) \times \hat{\vec{B}}(x)
$$

satisfies the equation of motion

$$
\frac{\partial}{\partial t} \frac{1}{2}\left(\hat{\vec{G}}(x)+\hat{\vec{G}}^{\dagger}(x)\right)=-\frac{1}{2}\left(\hat{\vec{L}}(x)+\hat{\vec{L}}^{\dagger}(x)\right)-\operatorname{div} \hat{\vec{\sigma}}(x) .
$$

In this expression, $\hat{\tilde{\sigma}}(x)$ is the Maxwell stress tensor density operator and $\hat{\vec{L}}(x)$ is the Lorentz force density operator as follows: 


$$
\begin{aligned}
& \hat{\sigma}^{i j}(x)=\frac{1}{8 \pi}\left(\hat{\vec{E}}^{2}(x) \delta_{i j}-\left(\hat{E}^{i}(x) \hat{E}^{j}(x)+\hat{E}^{j}(x) \hat{E}^{i}(x)\right)\right) \\
& +\frac{1}{8 \pi}\left(\hat{\vec{B}}^{2}(x) \delta_{i j}-\left(\hat{B}^{i}(x) \hat{B}^{j}(x)+\hat{B}^{j}(x) \hat{B}^{i}(x)\right)\right)
\end{aligned}
$$

and

$$
\begin{aligned}
& \hat{\vec{L}}(x)=\hat{\vec{L}}_{e}(x)+\sum_{a} \hat{\vec{L}}_{a}(x), \\
& \hat{\vec{L}}_{e}(x)=\hat{\vec{E}}(x) \hat{\rho}_{e}(x)+\frac{1}{c} \hat{\vec{j}}_{e}(x) \times \hat{\vec{B}}(x), \\
& \hat{\vec{L}}_{a}(x)=\hat{\vec{E}}(x) \hat{\rho}_{a}(x)+\frac{1}{c} \hat{\vec{j}}_{a}(x) \times \hat{\vec{B}}(x),
\end{aligned}
$$

where $\hat{\vec{L}}_{e}(x)$ is the electronic Lorentz force density operator and $\hat{\vec{L}}_{a}(x)$ is the Lorentz force density operator of $a$ 'th atomic nucleus.

It should be noted that $\hat{\vec{\sigma}}(x)$ is symmetric:

$$
\hat{\sigma}^{i j}(x)=\hat{\sigma}^{j i}(x)
$$

Next, the electronic kinetic momentum density operator $\hat{\vec{\Pi}}_{e}(\vec{r})$

$$
\hat{\vec{\Pi}}_{e}(x)=\frac{1}{2}\left(i \hbar \hat{\psi}^{\dagger}(x) \hat{\vec{D}}_{e}(x) \hat{\psi}(x)-i \hbar\left(\hat{\vec{D}}_{e}(x) \hat{\psi}(x)\right)^{\dagger} \cdot \hat{\psi}(x)\right),
$$

satisfies the equation of motion

$$
\frac{\partial}{\partial t} \hat{\vec{\Pi}}_{e}(x)=\hat{\vec{L}}_{e}(x)+\hat{\vec{\tau}}_{e}^{\Pi}(x) .
$$

Aside from the electronic Lorentz force density operator $\hat{\vec{L}}_{e}(x)$, the $\hat{\vec{\tau}}_{e}^{\Pi}(x)$ denotes the electronic tension density operator given as the divergence of the electronic stress tensor density operator $\hat{\vec{\tau}}_{e}^{\Pi}(x)$ as follows:

$$
\hat{\vec{\tau}}_{e}^{\Pi}(x)=\operatorname{div} \hat{\vec{\tau}}_{e}^{\Pi}(x),
$$

where 


$$
\begin{aligned}
& \hat{\tau}_{e}^{\Pi k}(x)=\frac{i \hbar c}{2}\left[\left(\hat{D}_{e l}(x) \hat{\psi}(x)\right)^{\dagger} \gamma^{0} \gamma^{\ell} \cdot \hat{D}_{e k}(x) \hat{\psi}(x)+\hat{\bar{\psi}}(x) \gamma^{\ell} \hat{D}_{e k}(x) \hat{D}_{e l}(x) \hat{\psi}(x)\right. \\
& \left.-\left(\hat{D}_{e k}(x) \hat{D}_{e l}(x) \hat{\psi}(x)\right)^{\dagger} \gamma^{0} \gamma^{\ell} \cdot \hat{\psi}(x)-\left(\hat{D}_{e k}(x) \hat{\psi}(x)\right)^{\dagger} \gamma^{0} \gamma^{\ell} \cdot \hat{D}_{e l}(x) \hat{\psi}(x)\right] \\
& -\frac{1}{c}\left(\hat{\vec{j}}_{e}(x) \times \vec{B}(x)\right)^{k}
\end{aligned}
$$

and

$$
\hat{\tau}_{e}^{\Pi k l}(x)=\frac{i \hbar c}{2}\left[\hat{\bar{\psi}}(x) \gamma^{\ell} \hat{D}_{e k}(x) \hat{\psi}(x)-\left(\hat{D}_{e k}(x) \hat{\psi}(x)\right)^{\dagger} \gamma^{0} \gamma^{\ell} \cdot \hat{\psi}(x)\right] .
$$

It should be noted that $\hat{\vec{\tau}}_{e}^{\Pi}(x)$ is Hermitean:

$$
\hat{\vec{\tau}}_{e}^{\Pi \dagger}(x)=\hat{\vec{\tau}}_{e}^{\Pi}(x) .
$$

Lastly, the kinetic momentum density operator $m_{a} \hat{\vec{v}}_{a}(x)$ of atomic nucleus $a$ satisfies the equation of motion

$$
\frac{\partial}{\partial t}\left(m_{a} \hat{\vec{v}}_{a}(x)\right)=\hat{\vec{L}}_{a}(x)+\hat{\vec{\tau}}_{a}^{s}(x) .
$$

Aside from the Lorentz force density operator $\hat{\vec{L}}_{a}(x)$, the $\hat{\vec{\tau}}_{a}^{S}(x)$ denotes the tension density operator given as the divergence of the stress tensor density operator $\hat{\vec{\tau}}_{a}^{S}(x)$ as follows:

$$
\hat{\vec{\tau}}_{a}^{s}(x)=\operatorname{div} \hat{\vec{\tau}}_{a}^{s}(x),
$$

where

$$
\begin{aligned}
& \hat{\tau}_{a}^{S k}(x)=\frac{\hbar^{2}}{4 m_{a}}\left[\hat{\chi}_{a}^{\dagger}(x) \hat{D}_{a k}(x) \hat{\vec{D}}_{a}^{2}(x) \hat{\chi}_{a}(x)+\left(\hat{D}_{a k}(x) \hat{\vec{D}}_{a}^{2}(x) \hat{\chi}_{a}(x)\right)^{\dagger} \cdot \hat{\chi}_{a}(x)\right. \\
& -\left(\hat{D}_{a k}(x) \hat{\chi}_{a}(x)\right)^{\dagger} \cdot \hat{\vec{D}}_{a}^{2}(x) \hat{\chi}_{a}(x)-\left(\hat{\vec{D}}_{a}^{2}(x) \hat{\chi}_{a}(x)\right)^{\dagger} \cdot \hat{D}_{a k}(x) \hat{\chi}_{a}(x) \\
& -\frac{1}{c}(\hat{\vec{j}}(x) \times \hat{\vec{B}}(x))^{k} \\
& \hat{\tau}_{a}^{S k l}(x)=\frac{\hbar^{2}}{4 m_{a}}\left[\hat{\chi}_{a}^{\dagger}(x) \hat{D}_{a k}(x) \hat{D}_{a l}(x) \hat{\chi}_{a}(x)+\left(\hat{D}_{a k}(x) \hat{D}_{a l}(x) \hat{\chi}_{a}(x)\right)^{\dagger} \cdot \hat{\chi}_{a}(x)\right. \\
& \left.-\left(\hat{D}_{a k}(x) \hat{\chi}_{a}(x)\right)^{\dagger} \cdot \hat{D}_{a l}(x) \hat{\chi}_{a}(x)-\left(\hat{D}_{a l}(x) \hat{\chi}_{a}(x)\right)^{\dagger} \cdot \hat{D}_{a k}(x) \hat{\chi}_{a}(x)\right]
\end{aligned}
$$


It should be noted that the stress tensor density operator $\hat{\hat{\tau}}_{a}^{s}(x)$ is Hermitean and symmetric:

$$
\begin{aligned}
& \hat{\vec{\tau}}_{a}^{S \dagger}(x)=\hat{\vec{\tau}}_{a}^{S}(x), \\
& \hat{\tau}_{a}^{S k l}(x)=\hat{\tau}_{a}^{S l k}(x) .
\end{aligned}
$$

As a whole, we obtain

$$
\begin{aligned}
& \frac{\partial}{\partial t} \hat{\vec{\Pi}}(x)=\hat{\vec{L}}(x)+\hat{\vec{\tau}}(x) \\
& =\hat{\vec{L}}(x)+\operatorname{div} \hat{\vec{\tau}}(x)
\end{aligned}
$$

where

$$
\begin{aligned}
& \hat{\vec{\Pi}}(x)=\hat{\vec{\Pi}}_{e}(x)+\sum_{a} m_{a} \hat{\vec{v}}_{a}(x), \\
& \hat{\vec{\tau}}(x)=\hat{\vec{\tau}}_{e}^{\Pi}(x)+\sum_{a} \hat{\vec{\tau}}_{a}^{S}(x), \\
& \hat{\vec{\tau}}(x)=\hat{\vec{\tau}}_{e}^{\Pi}(x)+\sum_{a} \hat{\vec{\tau}}_{a}^{s}(x) .
\end{aligned}
$$

Likewise, we obtain

$$
\frac{\partial}{\partial t}\left(\frac{1}{2}\left(\hat{\vec{G}}(x)+\hat{\vec{G}}^{\dagger}(x)\right)+\hat{\vec{\Pi}}(x)\right)=-\operatorname{div}(\hat{\vec{\sigma}}(x)-\hat{\vec{\tau}}(x)),
$$

which is the momentum conservation law of the Rigged QED system.

\section{Energy density}

The QED Hamiltonian density operator $\hat{H}_{\mathrm{QED}}(x)$ is composed of the Hamiltonian density operator of the electromagnetic field $\hat{H}_{\mathrm{EM}}(x)$ and the Dirac electronic Hamiltonian density operator $\hat{H}_{\text {Dirac }}(x)$ interacting with the electromagnetic field as follows [22]:

$$
\begin{aligned}
& \hat{H}_{\mathrm{QED}}(x)=\hat{H}_{\mathrm{EM}}(x)+\hat{H}_{\mathrm{Dirac}}(x), \\
& \hat{H}_{\mathrm{EM}}(x)=\hat{H}_{\gamma}(x)-\hat{A}_{0}(x) \hat{\rho}_{e}(x), \\
& \hat{H}_{\text {Dirac }}(x)=\hat{M}_{e}(x)+\hat{A}_{0}(x) \hat{\rho}_{e}(x),
\end{aligned}
$$


where $\hat{H}_{\gamma}(x)$ is the electromagnetic field energy density operator and $\hat{M}_{e}(x)$ is the electronic mass density operator:

$$
\begin{aligned}
& \hat{H}_{\gamma}(x)=\frac{1}{8 \pi}\left(\hat{\vec{E}}^{2}(x)+\hat{\vec{B}}^{2}(x)\right), \\
& \hat{M}_{e}(x)=c \hat{\bar{\psi}}(x)\left(-i \hbar \gamma^{k} \hat{D}_{e k}(x)+m_{e} c\right) \hat{\psi}(x),
\end{aligned}
$$

The electronic mass density operator $\hat{M}_{e}(x)$ may be written as the energy density operator of electron $\hat{H}_{e}(x)$ as follows:

$$
\hat{M}_{e}(x)=\hat{H}_{e}(x)
$$

Thus, the $\hat{H}_{\mathrm{QED}}(x)$ reduces from Eq. (3.1) to

$$
\hat{H}_{\mathrm{QED}}(x)=\hat{H}_{\gamma}(x)+\hat{H}_{e}(x) .
$$

The Rigged QED Hamiltonian density operator denoted as $\hat{H}_{\text {Rigged QED }}(x)$ is derived as follows:

$$
\hat{H}_{\text {Rigged QED }}(x)=\hat{H}_{\mathrm{QED}}(x)+\hat{H}_{\text {atom }}(x),
$$

where the energy density operator $\hat{H}_{\text {atom }}(x)$ of atomic nuclei interacting through the electromagnetic field and the electron field is added to $\hat{H}_{\mathrm{QED}}(x)$. The $\hat{H}_{\text {atom }}(x)$ is purely the kinetic energy density operator of atomic nuclei:

$$
\begin{aligned}
& \hat{H}_{\text {atom }}(x)=\sum_{a} \hat{T}_{a}(x), \\
& \hat{T}_{a}(x)=-\frac{\hbar^{2}}{2 m_{a}} \cdot \frac{1}{2}\left(\hat{\chi}_{a}^{\dagger}(x) \hat{\vec{D}}_{a}^{2}(x) \hat{\chi}_{a}(x)+\left(\hat{\vec{D}}_{a}^{2}(x) \hat{\chi}_{a}(x)\right)^{\dagger} \cdot \hat{\chi}_{a}(x)\right) .
\end{aligned}
$$

The energy flow is found as follows:

$$
\frac{\partial}{\partial t} \hat{H}_{\gamma}(x)=-c^{2} \operatorname{div} \frac{1}{2}\left(\hat{\vec{G}}(x)+\hat{\vec{G}}^{\dagger}(x)\right)-\frac{1}{2}(\hat{\vec{E}}(x) \bullet \hat{\vec{j}}(x)+\hat{\vec{j}}(x) \bullet \hat{\vec{E}}(x)),
$$




$$
\begin{aligned}
& \frac{\partial}{\partial t} \hat{H}_{e}(x)=-c^{2} \operatorname{div} \hat{\vec{\Pi}}_{e}(x)+\frac{1}{2}\left(\hat{\vec{E}}(x) \bullet \hat{\vec{j}}_{e}(x)+\hat{\vec{j}}_{e}(x) \bullet \hat{\vec{E}}(x)\right), \\
& \frac{\partial}{\partial t} \hat{H}_{\text {atom }}(x)=-\operatorname{div} \sum_{a} \hat{\vec{s}}_{a}(x)+\frac{1}{2}\left(\hat{\vec{E}}(x) \bullet \sum_{a} \hat{\vec{j}}_{a}(x)+\sum_{a} \hat{\vec{j}}_{a}(x) \bullet \hat{\vec{E}}(x)\right),
\end{aligned}
$$

with

$$
\hat{s}_{a}^{k}(x)=\frac{1}{2 i \hbar}\left(\frac{\hbar^{2}}{2 m_{a}}\right)\left(\begin{array}{l}
\left(\hat{D}_{a k}(x) \hat{\vec{D}}_{a}^{2}(x) \hat{\chi}_{a}(x)\right)^{\dagger} \cdot \hat{\chi}_{a}(x) \\
-\left(\hat{\vec{D}}_{a}^{2}(x) \hat{\chi}_{a}(x)\right)^{\dagger} \cdot \hat{D}_{a k}(x) \hat{\chi}_{a}(x) \\
+\left(\hat{D}_{a k}(x) \hat{\chi}_{a}(x)\right)^{\dagger} \cdot \hat{\vec{D}}_{a}^{2}(x) \hat{\chi}_{a}(x) \\
-\hat{\chi}_{a}^{\dagger}(x) \hat{D}_{a k}(x) \hat{\vec{D}}_{a}^{2}(x) \hat{\chi}_{a}(x)
\end{array}\right),
$$

leading to

$$
\frac{\partial}{\partial t} \hat{H}_{\text {Rigged QED }}(x)=-\operatorname{div}\left(c^{2} \frac{1}{2}\left(\hat{\vec{G}}(x)+\hat{\vec{G}}^{\dagger}(x)\right)+c^{2} \hat{\vec{\Pi}}_{e}(x)+\sum_{a} \hat{\vec{s}}_{a}(x)\right),
$$

which is the energy conservation law of the Rigged QED system.

The virial theorem has been found to be [7]:

$$
\begin{aligned}
& E_{\text {Rigged QED }}=\int d^{3} \vec{r}\left\langle\hat{H}_{\text {rigged QED }}(x)\right\rangle \\
& =\int d^{3} \vec{r}\left\langle m_{e} c^{2} \hat{\bar{\psi}}(x) \hat{\psi}(x)-\sum_{a} \hat{T}_{a}(x)\right\rangle
\end{aligned}
$$

which in the nonrelativistic limit becomes

$$
E_{\text {non-relativistic Rigged QED }}=-\int d^{3} \vec{r}\left\langle\sum_{\alpha} \hat{T}_{\alpha}(x)\right\rangle .
$$

It should be noted that the stress tensor density has the dimension of the energy density, namely energy per volume, since it has dimension of force per area. Indeed, the trace of the stress tensor density becomes

$$
\begin{aligned}
& \hat{\tau}_{e}^{\Pi k}(x) \\
& =\frac{c}{2}\left[-i \hbar \hat{\bar{\psi}}(x) \gamma^{k} \hat{D}_{e k}(x) \hat{\psi}(x)+i \hbar\left(\hat{D}_{e k}(x) \hat{\psi}(x)\right)^{\dagger} \gamma^{0} \gamma^{k} \cdot \hat{\psi}(x)\right], \\
& =\frac{1}{2}\left(\hat{M}_{e}(x)+\hat{M}_{e}^{\dagger}(x)\right)-m_{e} c^{2} \hat{\bar{\psi}}(x) \hat{\psi}(x)
\end{aligned}
$$




$$
\hat{\vec{\tau}}_{a \quad k}^{s \quad k}(x)=\frac{\hbar^{2}}{4 m_{a}}\left(\begin{array}{l}
-\hat{\chi}_{a}^{\dagger}(x) \hat{\vec{D}}_{a}^{2}(x) \hat{\chi}_{a}(x)-\left(\hat{\vec{D}}_{a}^{2}(x) \hat{\chi}_{a}(x)\right)^{\dagger} \cdot \hat{\chi}_{a}(x) \\
+2\left(\hat{D}_{a \ell}(x) \hat{\chi}_{a}(x)\right)^{\dagger} \cdot \hat{D}_{a \ell}(x) \hat{\chi}_{a}(x)
\end{array}\right) .
$$

This is equivalent to two times the kinetic energy density; in the nonrelativistic limit, the integral of the trace of the stress tensor density gives two times that of the kinetic energy density as follows:

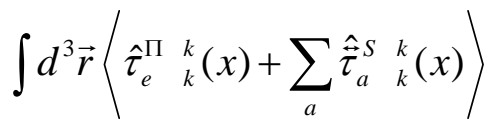

$$
\begin{aligned}
& \stackrel{\text { nonrelativistic limit }}{\longrightarrow} \int d^{3} \vec{r}\left\langle\sum_{\alpha} \hat{\vec{\tau}}_{\alpha}^{S} \quad k \quad k=\right\rangle=2 \int d^{3} \vec{r}\left\langle\sum_{\alpha} \hat{T}_{\alpha}(x)\right\rangle
\end{aligned}
$$

Hence, the stress tensor density gives a local picture of two times the kinetic energy density.

The eigenvalue is the principal stress and the eigenvector is the principal axis as summarized as follows:

$$
\begin{aligned}
& \left\langle\hat{\vec{\tau}}_{\alpha}^{S}(x)\right\rangle=\left(\begin{array}{ccc}
\tau_{\alpha}^{S 11}(x) & \tau_{\alpha}^{S 12}(x) & \tau_{\alpha}^{S 13}(x) \\
\tau_{\alpha}^{S 21}(x) & \tau_{\alpha}^{S 22}(x) & \tau_{\alpha}^{S 23}(x) \\
\tau_{\alpha}^{S 31}(x) & \tau_{\alpha}^{S 32}(x) & \tau_{\alpha}^{S 33}(x)
\end{array}\right) \\
& \underset{\operatorname{diag}}{\longrightarrow}\left(\begin{array}{ccc}
\tau_{\alpha}^{S 11}(x) & 0 & 0 \\
0 & \tau_{\alpha}^{S 22}(x) & 0 \\
0 & 0 & \tau_{\alpha}^{S 33}(x)
\end{array}\right) . \\
& \tau_{\alpha}^{S 11}(x) \leq \tau_{\alpha}^{S 22}(x) \leq \tau_{\alpha}^{S 33}(x) .
\end{aligned}
$$

We shall analyze the energy density of chemical bonds in the electronic stationary state.

Under external electromagnetic fields, charged particles can be accelerated by Lorentz force.

Dissipative force can bring about the stationary state of the charged particles. In quantum mechanics, energy eigenstate is another rule leading to the stationary state. As has been shown in this paper, tension of the quantum field theory can compensate the Lorentz force, which is the basic mechanism leading to the quantum mechanical stationary state: see equations (2.32), and (2.37).

The eigenvalue of the stress tensor density gives a measure of the kinetic energy density and the eigenvector, the principal axis, a measure of the direction of the bond. If the local principal stress is positive, it is called the tensile stress, while if it is negative, compressive. Since the metric tensor $g^{i j}$ has negative eigenvalues, $(-1,-1,-1)$, we should note that the compressive stress gives positive contribution to the kinetic energy density, while the tensile stress negative. This indicates the new picture of the local chemical interaction energy density. The atomic electron density exhibits positive kinetic energy density and forms the electronic drop region $R_{D}$ separated from the electronic 
atmosphere region $\mathrm{R}_{\mathrm{A}}$ by the interface $\mathrm{S}$, leading to the compressive stress. This tendency should of course be intact in between ionic species interactions. The situation should change dramatically for covalent bond formation, where a pair of electrons should be bound tightly, thereby creates tensile stress. Many systems exhibit such generic feature, which is called the spindle structure [5]. If in the electronic drop region $R_{D}$ in between a pair of atoms there crosses the Lagrange surface of the electronic tension, then the distribution of the electronic stresses and the principal axis is of great interest to study the character of the chemical bond. The local geometry is schematically shown in Figs. 2 and 3. For application to chemical reaction coordinates, we have also developed a concept of the interaction energy density [10-12].

\section{Local equilibrium in the stationary state \\ Repulsive electronic tension drives the quantum mechanical electronic "diffusion"}

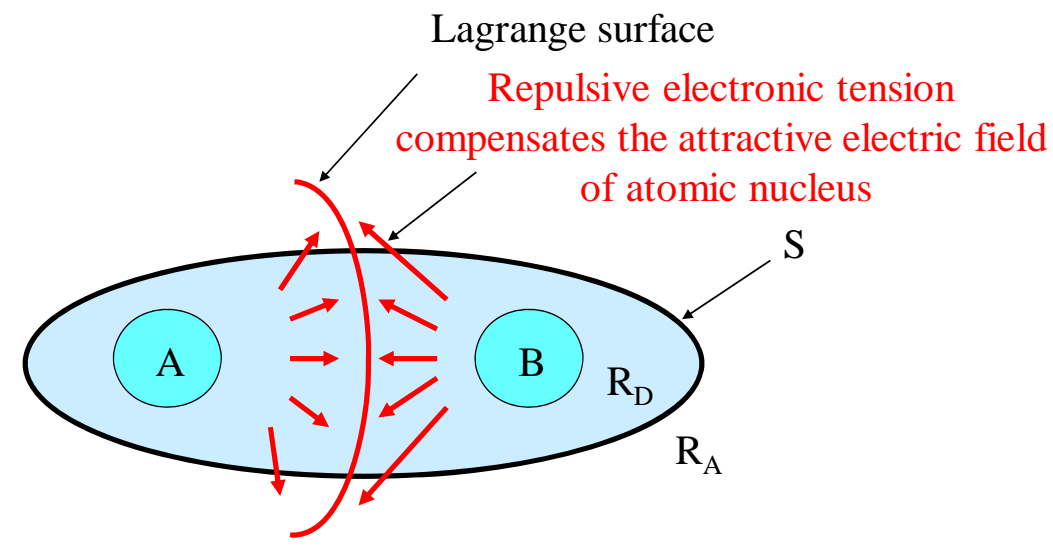

Fig. 2. Lagrange surface of the electronic tension in between a pair of atoms A and B. Also shown are the electronic drop region $R_{D}$ where the electronic kinetic energy density is positive, the electronic atmosphere region $\mathrm{R}_{\mathrm{A}}$ where the electronic kinetic energy density is negative, and the interface $\mathrm{S}$ which separates them. 


\section{Spindle structure of the covalent bond}

Electronic tensile stress binds a pair of the electronic drop regions $R_{D}$ 's where the compressive stress is predominant: covalent bond visualization!

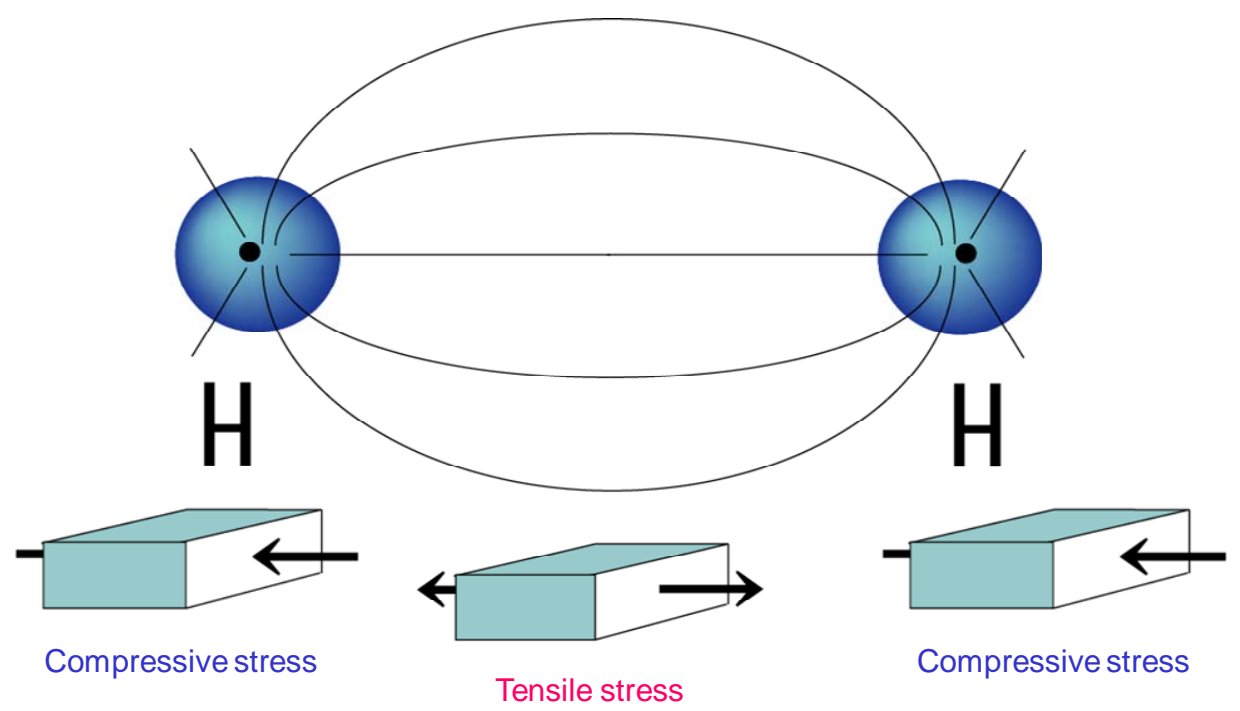

Fig. 3. Spindle structure scheme for covalent bond visualization in hydrogen molecule model. Electronic tensile stress binds a pair of the electronic drop regions $\mathrm{R}_{\mathrm{D}}$ 's where the compressive stress is predominant.

\section{Torque of spin angular momentum}

The angular momentum density operator $\hat{\vec{u}}(x)$ of the electromagnetic field defined as

$$
\hat{\vec{u}}(x)=\vec{r} \times \hat{\vec{G}}(x),
$$

satisfies the equation of motion

$$
\begin{aligned}
& \frac{\partial}{\partial t} \frac{1}{2}\left(\hat{\vec{u}}(x)+\hat{\vec{u}}^{\dagger}(x)\right)=-\vec{r} \times\left(\frac{1}{2}\left(\hat{\vec{L}}(x)+\hat{\vec{L}}^{\dagger}(x)\right)+\operatorname{div} \hat{\vec{\sigma}}(x)\right), \\
& =-\vec{r} \times \frac{1}{2}\left(\hat{\vec{L}}(x)+\hat{\vec{L}}^{\dagger}(x)\right)-\operatorname{div}(\vec{r} \times \hat{\vec{\sigma}}(x))
\end{aligned}
$$

where we have used the symmetric character of $\hat{\tilde{\sigma}}(x)$.

The electronic spin angular momentum density operator $\frac{1}{2} \hbar \hat{\vec{\sigma}}_{e}(x)$ with 


$$
\hat{\vec{\sigma}}_{e}(x)=\hat{\psi}^{\dagger}(x) \vec{\sigma} \hat{\psi}(x)
$$

satisfies the equation of motion

$$
\frac{\partial}{\partial t}\left(\frac{1}{2} \hbar \hat{\vec{\sigma}}_{e}(x)\right)=\hat{\vec{t}}_{e}(x)+\hat{\vec{\zeta}}_{e}(x)
$$

where $\hat{\vec{t}}_{e}(x)$ denotes the spin-torque density operator defined as [10]

$$
\hat{t}_{e}^{k}(x)=-\varepsilon_{l n k} \hat{\tau}_{e}^{\Pi \ell n}(x) .
$$

The $\hat{\vec{\zeta}}_{e}(x)$ denotes the zeta force density operator defined as

$$
\hat{\zeta}_{e}^{k}(x)=-c \partial_{k}\left(\hat{\bar{\psi}}(x) \gamma^{k} \frac{1}{2} \hbar \sigma^{k} \hat{\psi}(x)\right) ; \text { no sum over k. }
$$

The alternative form is obtained as follows:

$$
\begin{aligned}
& \hat{\zeta}_{e}^{k}(x)=-\partial_{k} \hat{\phi}_{5}(x), \\
& \hat{\phi}_{5}(x)=\frac{\hbar}{2 Z_{e} e} \hat{j}_{5}^{0}(x),
\end{aligned}
$$

where $\hat{j}_{5}^{0}(x)$ denotes the zeroth component of the chiral current density operator

$$
\begin{aligned}
& \hat{j}_{5}^{\mu}(x)=c Z_{e} e \hat{\bar{\psi}}(x) \gamma^{\mu} \gamma_{5} \hat{\psi}(x), \\
& \gamma_{5}=i \gamma^{0} \gamma^{1} \gamma^{2} \gamma^{3} .
\end{aligned}
$$

The electronic orbital angular momentum density operator $\hat{\vec{\ell}}_{e}(x)$ defined as

$$
\hat{\vec{\ell}}_{e}(x)=\vec{r} \times \hat{\vec{\Pi}}_{e}(x),
$$

satisfies the equation of motion

$$
\begin{aligned}
& \frac{\partial}{\partial t} \hat{\vec{\ell}}_{e}(x)=\vec{r} \times\left(\hat{\vec{L}}_{e}(x)+\operatorname{div} \hat{\vec{\tau}}_{e}^{\Pi}(x)\right) \\
& =\vec{r} \times \hat{\vec{L}}_{e}(x)+\operatorname{div}\left(\vec{r} \times \hat{\vec{\tau}}_{e}^{\Pi}(x)\right)-\hat{\vec{t}}_{e}(x)
\end{aligned} .
$$

Sum of equations (4.4) and (4.12) leads to 


$$
\begin{aligned}
& \frac{\partial}{\partial t}\left(\hat{\vec{\ell}}_{e}(x)+\frac{1}{2} \hbar \hat{\vec{\sigma}}_{e}(x)\right)=\vec{r} \times\left(\hat{\overrightarrow{\vec{L}}}_{e}(x)+\operatorname{div} \hat{\vec{\tau}}_{e}^{\Pi}(x)\right)+\hat{\vec{t}}_{e}(x)+\hat{\vec{\zeta}}_{e}(x) \\
& =\vec{r} \times \hat{\vec{L}}_{e}(x)+\operatorname{div}\left(\vec{r} \times \hat{\vec{\tau}}_{e}^{\Pi}(x)\right)+\hat{\vec{\zeta}}_{e}(x)
\end{aligned}
$$

The $a$ 'th nuclear orbital angular momentum density operator $\hat{\vec{\ell}}_{a}(x)$ defined as

$$
\hat{\vec{\ell}}_{a}(x)=\vec{r} \times m_{a} \hat{\vec{V}}_{a}(x)
$$

satisfies the equation of motion:

$$
\frac{\partial}{\partial t} \hat{\vec{\ell}}_{a}(x)=\vec{r} \times \hat{\vec{L}}_{a}(x)+\operatorname{div}\left(\vec{r} \times \hat{\vec{\tau}}_{a}^{s}(x)\right)
$$

where we have used the symmetric character of $\hat{\vec{\tau}}_{a}^{S}(x)$.

For the angular momentum as a whole, we have

$$
\begin{aligned}
& \frac{\partial}{\partial t}\left(\hat{\vec{\ell}}_{e}(x)+\frac{1}{2} \hbar \hat{\vec{\sigma}}_{e}(x)+\sum_{a} \hat{\vec{\ell}}_{a}(x)\right)=\vec{r} \times \hat{\vec{L}}(x)+\vec{r} \times \operatorname{div} \hat{\vec{\tau}}(\vec{r})+\hat{\vec{t}}_{e}(x)+\hat{\vec{\zeta}}_{e}(x), \\
& =\vec{r} \times \hat{\vec{L}}(x)+\operatorname{div}(\vec{r} \times \hat{\vec{\tau}}(x))+\hat{\vec{\zeta}}_{e}(x)
\end{aligned}
$$

and if the $\hat{\vec{u}}(x)$ is further added to, we finally obtain

$$
\begin{aligned}
& \frac{\partial}{\partial t}\left(\frac{1}{2}\left(\hat{\vec{u}}(x)+\hat{\vec{u}}^{\dagger}(x)\right)+\hat{\vec{\ell}}_{e}(x)+\frac{1}{2} \hbar \hat{\vec{\sigma}}_{e}(x)+\sum_{a} \hat{\overrightarrow{\hat{\ell}}}_{a}(x)\right) \\
& =-\vec{r} \times \operatorname{div}(\hat{\vec{\sigma}}(x)-\hat{\vec{\tau}}(x))+\hat{\vec{t}}_{e}(x)+\hat{\vec{\zeta}}_{e}(x) \\
& =-\operatorname{div}(\vec{r} \times(\hat{\vec{\sigma}}(x)-\hat{\vec{\tau}}(x)))+\hat{\vec{\zeta}}_{e}(x)
\end{aligned},
$$

which is the angular momentum conservation law of the Rigged QED system.

Electronic spin can therefore be accelerated by the torque derived by the stress tensor, which is also compensated by the zeta force, which is another basic mechanism leading to the stationary state of electronic spin.

It should be noted that Pauli Hamiltonian gives equation of motion of electronic spin: see, e.g., equation (11.155) of Jackson's textbook on classical electrodynamics [23]. The BMT (Bargmann-Michel-Telegdi) equation and Thomas precession are also the textbook matters. Our present result incorporates all of them in a closed form plus the field theoretical compensation mechanism leading to the stationary state of electronic spin. 


\section{Effective charge density of electromigration}

\subsection{Electromagnetic energy density in magnetodielectric media}

In the Rigged QED theory, the phenomenological interaction of a system A and its environment background medium $\mathrm{M}$ is tractable using regional charge and current densities. It is easily generalized for a system A embedded in the surrounding medium M. For phenomenological force concepts in magnetodielectric medium such as chemical reaction systems in condensed phase, we may usually rely on a classical analogy of parallel-plate capacitor filled with a dielectric as shown in Fig. 4.

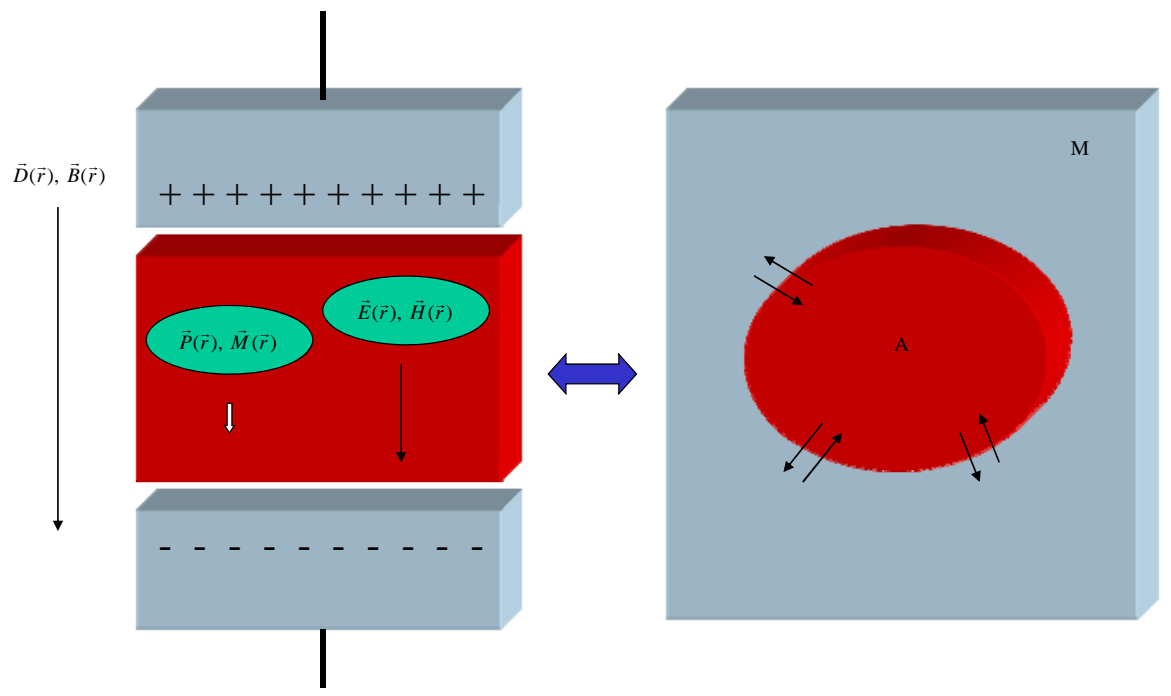

Fig. 4. Parallel-plate capacitor filled with a dielectric: a phenomenological model of a chemical reaction system A embedded in an environmental background medium $\mathrm{M}$.

The corresponding gauge potentials are the regional integrals of the charge and transversal current densities, defined as follows [7]:

$$
\begin{aligned}
& \hat{A}_{0_{\mathrm{A}}}(c t, \vec{r})=\int_{\mathrm{A}} d^{3} \vec{s} \frac{\hat{\rho}(c t, \vec{s})}{|\vec{r}-\vec{s}|}, \\
& \hat{A}_{0_{\mathrm{M}}}(c t, \vec{r})=\int_{\mathrm{M}} d^{3} \vec{s} \frac{\hat{\rho}(c t, \vec{s})}{|\vec{r}-\vec{s}|},
\end{aligned}
$$

and 


$$
\begin{aligned}
& \hat{\vec{A}}_{\mathrm{A}}(c t, \vec{r})=\frac{1}{c} \int_{\mathrm{A}} d^{3} \vec{s} \frac{\hat{\vec{j}}_{\mathrm{T}}(c u, \vec{s})}{|\vec{r}-\vec{s}|}, \\
& \hat{\vec{A}}_{\mathrm{M}}(c t, \vec{r})=\frac{1}{c} \int_{\mathrm{M}} d^{3} \vec{s} \frac{\hat{\vec{j}}_{\mathrm{T}}(c u, \vec{s})}{|\vec{r}-\vec{s}|},
\end{aligned}
$$

where the subscript A or $\mathrm{M}$ of the integral sign denotes the regional integrals confined to the region A or M, respectively, and where $u=t-\frac{|\vec{r}-\vec{s}|}{c}$.

Since the regions $\mathrm{A}$ and $\mathrm{M}$ altogether span the whole space, we have

$$
\begin{aligned}
& \hat{A}_{0}(x)=\hat{A}_{0_{\mathrm{A}}}(x)+\hat{A}_{0_{\mathrm{M}}}(x), \\
& \hat{\vec{A}}(x)=\hat{\vec{A}}_{\mathrm{A}}(x)+\hat{\vec{A}}_{\mathrm{M}}(x)+\hat{\vec{A}}_{\text {radiation }}(x) .
\end{aligned}
$$

The electric field $\hat{\vec{E}}(x)$ is decomposed into the electric displacement $\hat{\vec{D}}(x)$ of the medium M and the polarization $\hat{\vec{P}}(x)$ of the system A, defined respectively as

$$
\begin{aligned}
& \hat{\vec{D}}(x)=-\operatorname{grad} \hat{A}_{0_{\mathrm{M}}}(x)-\frac{1}{c} \frac{\partial}{\partial t} \hat{\vec{A}}_{\mathrm{M}}(x), \\
& \hat{\vec{P}}(x)=\frac{1}{4 \pi} \operatorname{grad} \hat{A}_{0_{\mathrm{A}}}(x)+\frac{1}{4 \pi c} \frac{\partial}{\partial t} \hat{\vec{A}}_{\mathrm{A}}(x),
\end{aligned}
$$

so that we have

$$
\begin{aligned}
& \hat{\vec{E}}(x)=-\operatorname{grad} \hat{A}_{0}(x)-\frac{1}{c} \frac{\partial}{\partial t} \hat{\vec{A}}(x) \\
& =\hat{\vec{D}}(x)-4 \pi \hat{\vec{P}}(x)-\frac{1}{c} \frac{\partial}{\partial t} \hat{\vec{A}}_{\text {radiation }}(x)
\end{aligned}
$$

It should be noted that the $\hat{\vec{P}}(x)$ generalizes the usual dipole field.

Likewise, let the magnetic field $\hat{\vec{H}}(x)$ of the medium $M$ and the magnetization $\hat{\vec{M}}(x)$ of the system A be defined respectively as

$$
\begin{aligned}
& \hat{\vec{H}}_{\mathrm{M}}(x)=\operatorname{rot} \hat{\vec{A}}_{\mathrm{M}}(x), \\
& \hat{\vec{M}}(x)=\frac{1}{4 \pi} \operatorname{rot} \hat{\vec{A}}_{\mathrm{A}}(x),
\end{aligned}
$$


then we have

$$
\begin{aligned}
& \hat{\vec{B}}(x)=\operatorname{rot} \hat{\vec{A}}(x) \\
& =\hat{\vec{H}}(x)+4 \pi \hat{\vec{M}}(x) \text {, with } \\
& \hat{\vec{H}}(x)=\hat{\vec{H}}_{\mathrm{M}}(x)+\operatorname{rot} \hat{\vec{A}}_{\text {radiation }}(x)
\end{aligned}
$$

The regional charge densities are then represented respectively as

$$
\begin{aligned}
& \hat{\rho}_{\mathrm{A}}(x)=-\frac{1}{4 \pi} \Delta \hat{A}_{0_{\mathrm{A}}}(x), \\
& \hat{\rho}_{\mathrm{M}}(x)=-\frac{1}{4 \pi} \Delta \hat{A}_{0_{\mathrm{M}}}(x),
\end{aligned}
$$

with the Laplacian

$$
\Delta=\frac{\partial^{2}}{\partial x^{2}}+\frac{\partial^{2}}{\partial y^{2}}+\frac{\partial^{2}}{\partial z^{2}},
$$

and hence

$$
\hat{\rho}(x)=\hat{\rho}_{\mathrm{A}}(x)+\hat{\rho}_{\mathrm{M}}(x) .
$$

Likewise, the regional charge current densities are represented as

$$
\begin{aligned}
& \hat{\vec{j}}_{\mathrm{A}}(x)=\frac{c}{4 \pi}\left(\frac{1}{c} \operatorname{grad} \frac{\partial}{\partial t} \hat{A}_{0_{\mathrm{A}}}(x)+\square \hat{\vec{A}}_{\mathrm{A}}(x)\right), \\
& =\frac{\partial}{\partial t} \hat{\vec{P}}(x)+c \operatorname{rot} \hat{\vec{M}}(x) \\
& \hat{\vec{j}}_{\mathrm{M}}(x)=\frac{c}{4 \pi}\left(\frac{1}{c} \operatorname{grad} \frac{\partial}{\partial t} \hat{A}_{0_{\mathrm{M}}}(x)+\square \hat{\vec{A}}_{\mathrm{M}}(x)\right),
\end{aligned}
$$

with the D'Alembercian

$$
\square=\frac{1}{c^{2}} \frac{\partial^{2}}{\partial t^{2}}-\Delta,
$$

and hence

$$
\begin{aligned}
& \hat{\vec{j}}(x)=\hat{\vec{j}}_{\mathrm{A}}(x)+\hat{\vec{j}}_{\mathrm{M}}(x) \\
& =\frac{\partial}{\partial t} \hat{\vec{P}}(x)+\operatorname{crot} \hat{\vec{M}}(x)+\hat{\vec{j}}_{\mathrm{M}}(x)
\end{aligned} .
$$

The regional decomposition of the longitudinal and transversal components of the current densities are represented as follows:

$$
\hat{\vec{j}}(x)=\hat{\vec{j}}_{\mathrm{L}}(x)+\hat{\vec{j}}_{\mathrm{T}}(x),
$$

with

$$
\hat{\vec{j}}_{\mathrm{L}}(x)=\hat{\vec{j}}_{\mathrm{L}_{\mathrm{A}}}(x)+\hat{\vec{j}}_{\mathrm{L}_{\mathrm{M}}}(x),
$$




$$
\hat{\vec{j}}_{\mathrm{T}}(x)=\hat{\vec{j}}_{\mathrm{T}_{\mathrm{A}}}(x)+\hat{\vec{j}}_{\mathrm{T}_{\mathrm{M}}}(x) \text {, }
$$

where

$$
\begin{aligned}
& \hat{\vec{j}}_{\mathrm{L}_{\mathrm{A}}}(x)=\frac{c}{4 \pi} \cdot \frac{1}{c} \operatorname{grad} \frac{\partial}{\partial t} \hat{A}_{\mathrm{O}_{\mathrm{A}}}(x), \\
& \hat{\vec{j}}_{\mathrm{L}_{\mathrm{M}}}(x)=\frac{c}{4 \pi} \cdot \frac{1}{c} \operatorname{grad} \frac{\partial}{\partial t} \hat{A}_{\mathrm{M}}(x), \\
& \hat{\vec{j}}_{\mathrm{T}_{\mathrm{A}}}(x)=\frac{c}{4 \pi} \cdot \square \hat{\vec{A}}_{\mathrm{A}}(x), \\
& \hat{\vec{j}}_{\mathrm{T}_{\mathrm{M}}}(x)=\frac{c}{4 \pi} \cdot \square \hat{\vec{A}}_{\mathrm{M}}(x) .
\end{aligned}
$$

Using Eqs. (5.24)-(5.27), we have the alternative forms of Eqs. (5.17) and (5.18) respectively as

$$
\begin{aligned}
& \hat{\vec{j}}_{\mathrm{A}}(x)=\hat{\vec{j}}_{\mathrm{L}_{\mathrm{A}}}(x)+\hat{\vec{j}}_{\mathrm{T}_{\mathrm{A}}}(x), \\
& \hat{\vec{j}}_{\mathrm{M}}(x)=\hat{\vec{j}}_{\mathrm{L}_{\mathrm{M}}}(x)+\hat{\vec{j}}_{\mathrm{T}_{\mathrm{M}}}(x) .
\end{aligned}
$$

The linear response properties of the system A under the interaction with the environment medium M may formally be represented with obvious notation as follows:

$$
\begin{aligned}
& \hat{\vec{P}}(x)=\hat{\vec{\alpha}}(x)\left(\hat{\vec{D}}(x)-\frac{1}{c} \frac{\partial}{\partial t} \hat{\overrightarrow{\vec{A}}}_{\text {radiation }}(x)\right) \\
& =\hat{\vec{\chi}}_{e}(x) \hat{\vec{E}}(x) \text {, and } \\
& \hat{\vec{M}}(x)=\hat{\vec{\chi}}_{m}(x) \hat{\vec{H}}(x) \\
& \hat{\vec{D}}(x)-\frac{1}{c} \frac{\partial}{\partial t} \hat{\vec{A}}_{\text {radiation }}(x)=\left(1+4 \pi \hat{\vec{\chi}}_{e}(x)\right) \hat{\vec{E}}(x) \\
& =\frac{1}{1-4 \pi \hat{\vec{\alpha}}(x)} \hat{\vec{E}}(x) \\
& =\hat{\vec{\varepsilon}}(x) \hat{\vec{E}}(x) \\
& \hat{\vec{B}}(x)=\left(1+4 \pi \hat{\vec{\chi}}_{m}(x)\right) \hat{\vec{H}}(x), \\
& =\hat{\vec{\mu}}(x) \hat{\vec{H}}(x) \\
& \text { and }
\end{aligned}
$$




$$
\begin{aligned}
& \hat{\vec{j}}(x)=\hat{\vec{\sigma}}_{\text {ext }}(x)\left(\hat{\vec{D}}(x)-\frac{1}{c} \frac{\partial}{\partial t} \hat{\vec{A}}_{\text {radiation }}(x)\right) \\
& =\hat{\vec{\sigma}}_{\text {ext }}(x) \hat{\vec{\varepsilon}}(x) \hat{\vec{E}}(x) \\
& =\hat{\vec{\sigma}}_{\text {int }}(x) \hat{\vec{E}}(x)
\end{aligned}
$$

\subsection{Effective charge number of electromigration}

Electromigration is the phenomena of nuclear current induced by electric current in condensed phase [24]. The nuclei accept diffusive force from the surrounding medium over and above the Lorentz force [25]. In our model, the tension is the origin of the medium effects [9].

The linear response of the force defines the effective charge number tensor density operator $\hat{Z}_{\alpha}^{*}(x)$ of $\alpha$ 'th charged particle as

$$
\hat{\vec{Z}}_{\alpha}^{*}(x)\left(\hat{\vec{D}}(x)-\frac{1}{c} \frac{\partial}{\partial t} \hat{\vec{A}}_{\text {radiation }}(x)\right) e \hat{N}_{\alpha}(x)+\frac{1}{c} \hat{\vec{j}}_{\alpha}(x) \times \hat{\vec{B}}(x)=\hat{\vec{L}}_{\alpha}(x)+\hat{\vec{\tau}}_{\alpha}^{s}(x) .
$$

Since the right hand side of this equation is

$$
\hat{\vec{L}}_{\alpha}(x)+\hat{\vec{\tau}}_{\alpha}^{S}(x)=\hat{\vec{E}}(x) \hat{\rho}_{\alpha}(x)+\frac{1}{c} \hat{\vec{j}}_{\alpha}(x) \times \hat{\vec{B}}(x)+\hat{\vec{\tau}}_{\alpha}^{S}(x),
$$

we then conclude

$$
\begin{aligned}
& \hat{\vec{Z}}_{\alpha}^{*}(x)\left(\hat{\vec{D}}(x)-\frac{1}{c} \frac{\partial}{\partial t} \hat{\vec{A}}_{\text {radiation }}(x)\right) e \hat{N}_{\alpha}(x)=\hat{\vec{E}}(x) \hat{\rho}_{\alpha}(x)+\hat{\vec{\tau}}_{\alpha}^{S}(x) \\
& =\left(\hat{\vec{D}}(x)-4 \pi \hat{\vec{P}}(x)-\frac{1}{c} \frac{\partial}{\partial t} \hat{\vec{A}}_{\text {radiation }}(x)\right) Z_{\alpha} e \hat{N}_{\alpha}(x)+\hat{\vec{\tau}}_{\alpha}^{S}(x) \\
& =\left(\hat{\vec{D}}(x)-\frac{1}{c} \frac{\partial}{\partial t} \hat{\vec{A}}_{\text {radiation }}(x)\right) Z_{\alpha} e \hat{N}_{\alpha}(x)-4 \pi \hat{\vec{P}}(x) Z_{\alpha} e \hat{N}_{\alpha}(x)+\hat{\vec{\tau}}_{\alpha}^{S}(x)
\end{aligned}
$$

Now we define

$$
\begin{aligned}
& \hat{\vec{Z}}_{\alpha}^{*}(x)=Z_{\alpha}+\hat{\vec{Z}}_{\alpha \text { wind }}(x), \\
& \hat{\vec{Z}}_{\alpha \text { wind }}(x)=\hat{\vec{Z}}_{\alpha \text { static wind }}(x)+\hat{\vec{Z}}_{\alpha \text { dynamic wind }}(x),
\end{aligned}
$$

and we conclude

$$
\begin{aligned}
& \hat{\vec{Z}}_{\alpha \text { static wind }}(x)=-4 \pi Z_{\alpha} \hat{\vec{\alpha}}(x), \\
& \hat{\vec{Z}}_{\alpha \text { dynamic wind }}(x)\left(\hat{\vec{D}}(x)-\frac{1}{c} \frac{\partial}{\partial t} \hat{\vec{A}}_{\text {radiation }}(x)\right) e \hat{N}_{\alpha}(x)=\hat{\vec{\tau}}_{\alpha}^{s}(x) .
\end{aligned}
$$


It should be noted that the formulation for $\alpha=e$ dictates the case of electronic conduction. In this case, the usual textbook approach demonstrates the medium effect as the dissipasive force against Lorentz force: see, e.g., Eq. (1.16) of Ashcroft-Mermin's textbook on solid state physics [26]. In our present result, the dissipative force emerges from the tension density as the field theoretical force density compensating the Lorentz force density.

\section{Example}

We shall demonstrate the energy density concept using hydrogen. We have already published the $H$ atom's $R_{D}, R_{A}$ and $S$ [4-8] and the spindle structure for covalent $H_{2}$ bond formation [5] as schematically shown in Figs.2 and 3. The density property of spin torque may here be added to. Since the electric field of proton is spherically symmetric, there appears no spin torque density for the ground and excited states nor the zeta force density. The sum of the two densities should then of

course zero, $\vec{t}_{e}(\vec{r})+\vec{\zeta}_{e}(\vec{r})=0$, at each point in space as it should be for the stationary state, Eq.(4.4).

Another example with chemical reaction of hydrogen economy may also be added to.

Recently, hydrogen-storage carbon materials for fuel cell applications have received much attention and the basic reaction mechanism has not been thoroughly known yet [27-42]. Since there should be certain energy barrier for $\mathrm{H}$ atom adsorption on graphene sheet $[29,30]$, our idea here is the charge transfer from hetero atoms such as $\mathrm{Al}$ may enhance the deformation of graphene which may then enhance stabilization of $\mathrm{H}$ atom on the graphene surface. We have performed $a b$ initio molecular-dynamics (MD) simulations using restricted Hartree-Fock (RHF) electronic wave function expanded by $6-31+\mathrm{G}^{*}$ basis set for $\mathrm{Al}$ and Dunning-Huzinaga full double zeta type ones for $\mathrm{H}$ and $\mathrm{C}$ for dynamic behaviors of $\mathrm{H}$ atoms on model graphene sheet with $\mathrm{Al}$ atom [43]. We shall present here some of the preliminary results for demonstration of the energy density concept. 


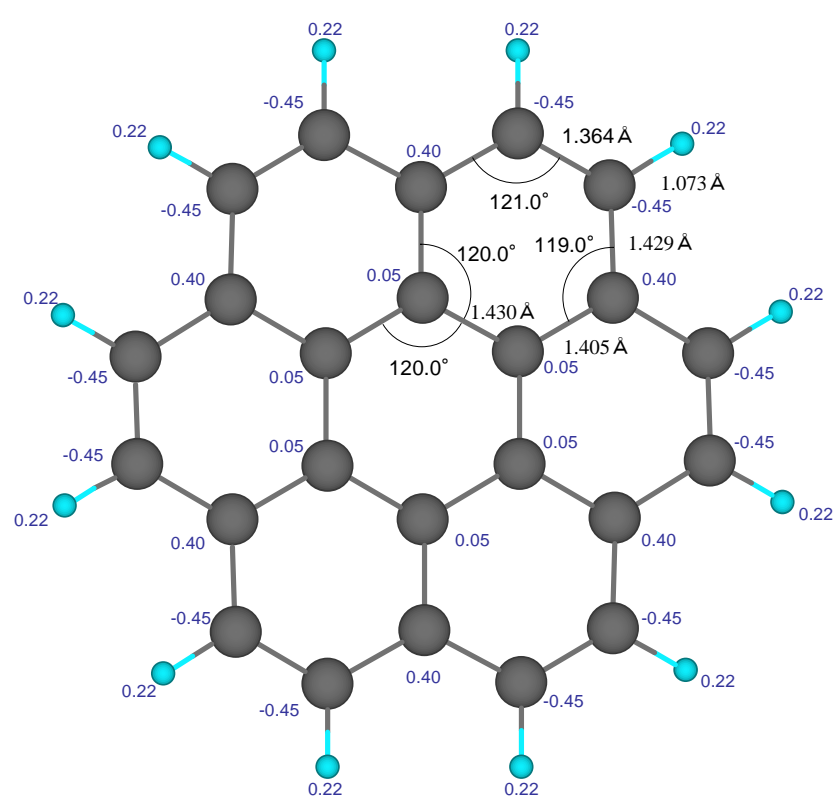

(a)

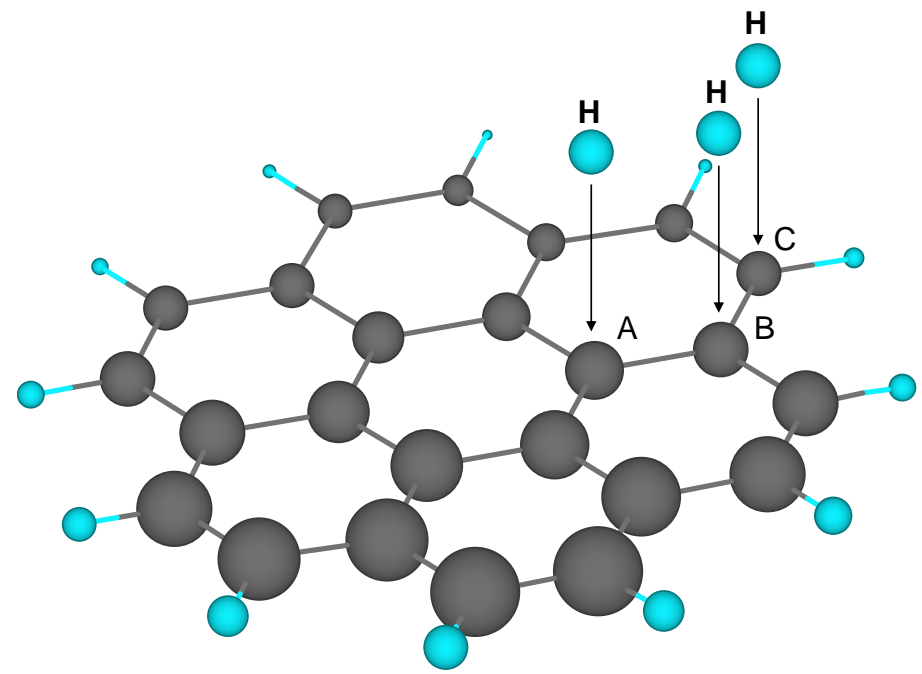

(b)

Fig. 5. Model graphene sheet (a) the optimized structure with Mulliken charges, and (b) the attacking sites $\mathrm{A}, \mathrm{B}$, and $\mathrm{C}$ by $\mathrm{H}$. 
We have shown in Fig. 5 (a) the optimized model graphene sheet with Mulliken charges, and (b) the three attacking sites $\mathrm{A}, \mathrm{B}$, and $\mathrm{C}$ by $\mathrm{H}$ on it.
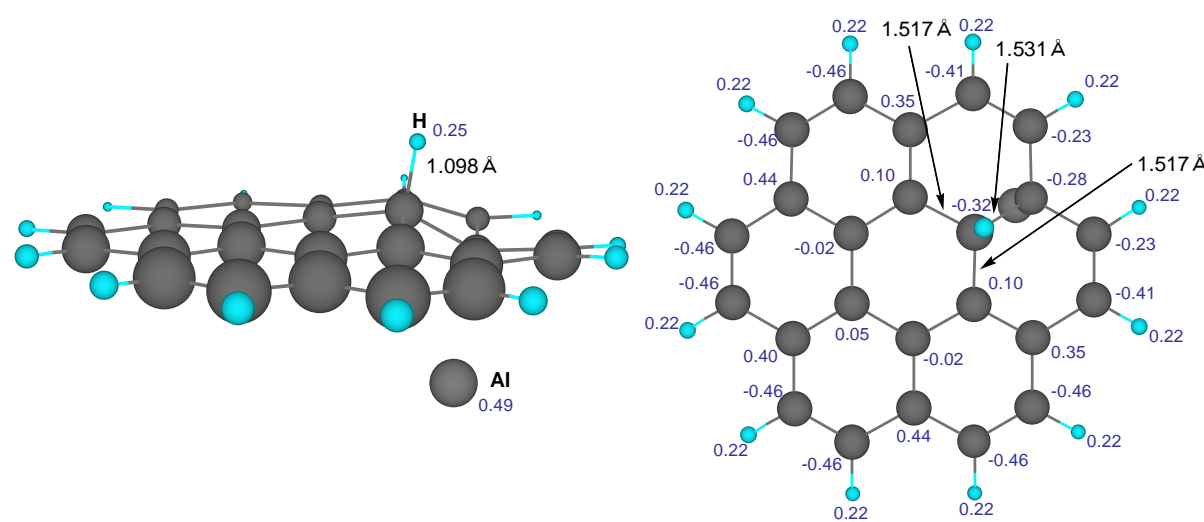

B.E. $=0.920 \mathrm{eV}$

\section{A-site optimized structure}

(a)

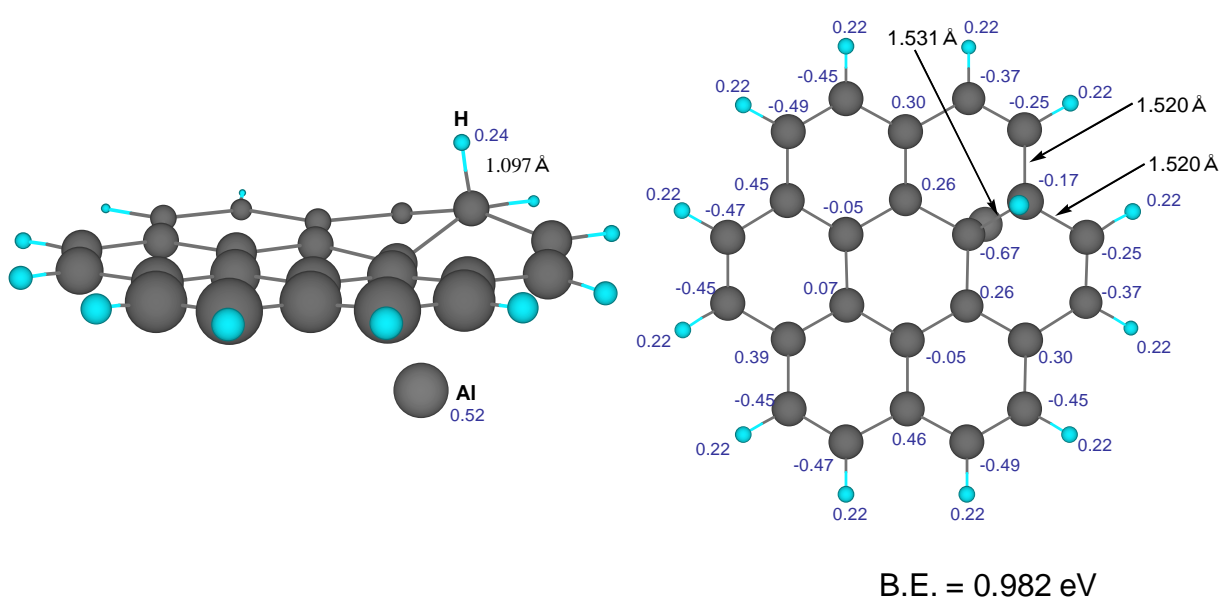

\section{B-site optimized structure}

(b) 

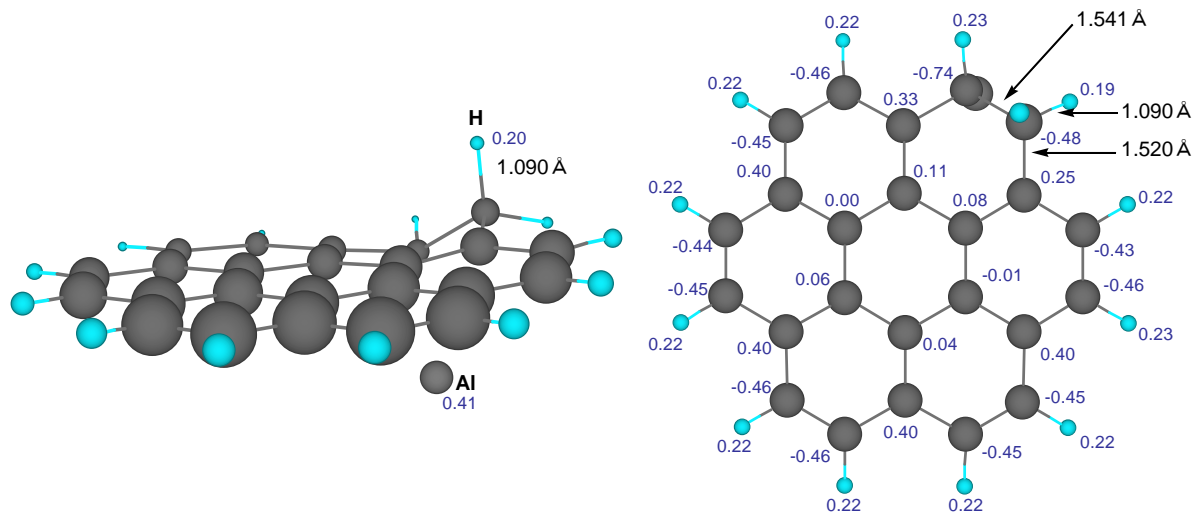

B.E. $=2.357 \mathrm{eV}$

\section{C-site optimized structure}

(c)

Fig. 6. Three of various local minima corresponding to (a) A, (b) B, and (c) C attacking sites by $\mathrm{H}$ in Fig. 5 with the binding energies (B.E.'s). The $\mathrm{Al}$ atom situated at the back side of the graphene sheet stabilizes the adducts through charge transfer.

In Fig. 6 are shown the three of various local minima corresponding to these sites with the binding energies (B.E.'s), where the $\mathrm{Al}$ atom situated at the opposite side of the graphene sheet stabilizes the adducts through charge transfer. 
(a)

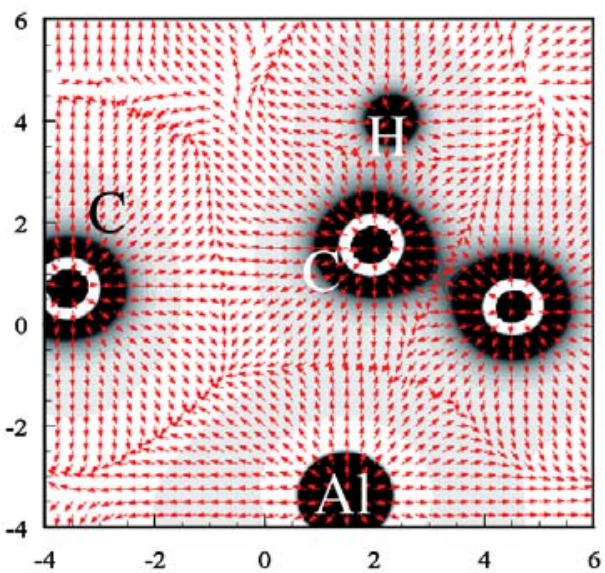

(b)

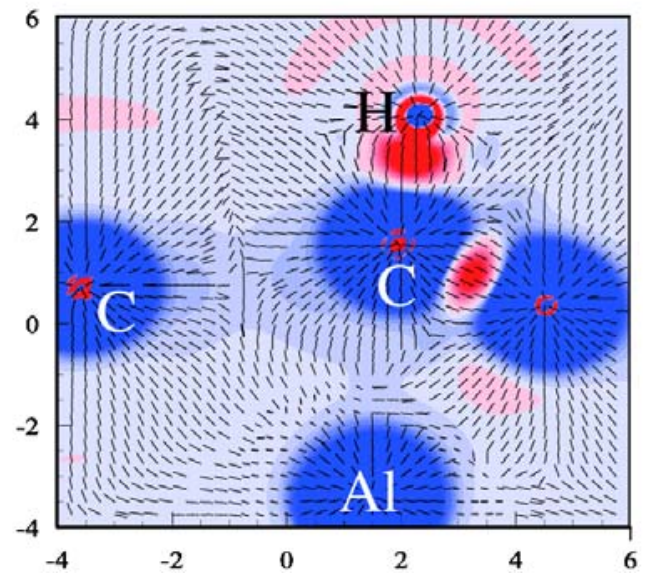

Fig. 7. Electronic energy densities on a cross section of a snapshot in the MD simulation. (a) the positive kinetic energy density in grey region and the tension density in red arrow, and (b) the $3^{\text {rd }}$ eigenvalue of the stress tensor density in red for positive "tensile" stress, while in blue negative "compressive" stress, and the eigenvector in black line. The axes of ordinate and abscissa are labeled in Bohr.

Fig. 7 shows a snapshot of electron energy density plots in the MD simulation drawn by Molecular Regional DFT program package developed in our laboratory [44]. In this snapshot the bond length between the $\mathrm{H}$ and the nearest $\mathrm{C}$ atom is $1.03 \AA$ A . The kinetic energy density in Fig. 7 (a) represents the charge transfer through the merged $\mathrm{R}_{\mathrm{D}}$ between $\mathrm{H}$ and $\mathrm{Al}$ atoms and the graphene sheet. The tension density represents the force which help the $\mathrm{sp}^{2}$ hybrid orbital change to $\mathrm{sp}^{3}$ stabilizing the floppy surface of the graphene sheet. The stress tensor density in Fig. 7 (b) shows the characteristic chemical bonding properties. The stress tensor density between the $\mathrm{H}$ and $\mathrm{C}$ atoms represents positive "tensile" stress and the "spindle structure" is formed between them. This dictates the covalent bond formation and hence they are tightly bound with each other. On the other hand, the region between the $\mathrm{Al}$ and $\mathrm{C}$ atoms represents negative "compressive" stress. This corresponds to the situation that the $\mathrm{Al}$ atom moves with no tightly covalent trapping points on the graphene sheet.

Detailed discussions including the dynamics with the spin torque for hydrogen storage processes under electric stresses of the external electromagnetic fields in the surrounding medium 
should be of considerable interest, and will be published elsewhere.

\section{Conclusion}

Under external source of electromagnetic fields, charged particles can be accelerated by Lorentz force. Dissipative force can make the state of the charged particles stationary. In quantum mechanics, energy eigenstate is another rule of the stationary state. In this paper, tension density of quantum field theory is formulated in such a way that it can compensate the Lorentz force density at any point of space-time. This formulation can give mechanical description of local equilibrium leading to the quantum mechanical stationary state. The tension density is given by the divergence of stress tensor density. Electronic spin can be accelerated by torque density derived from the stress tensor density. The torque density can be compensated by a force density, called the zeta force density, which is another basic mechanism leading to the stationary state of the spinning motion of electron. The external effect for chemical reaction systems is realized where a chemical reaction system A embedded in the environmental medium $\mathrm{M}$ is modeled as a parallel-plate capacitor filled with a dielectric. The vibronic interaction that goes beyond the adiabatic approximation has been incorporated as well as the electronic spin-dependent interaction.

\section{Acknowledgments}

This work has been supported in part by a Gant-in-Aid from the Ministry of Education, Culture, Science and Technology of Japan, for which we express our gratitude.

\section{APPENDIX A}

\section{A.1. The energy density in the non-Abelian gauge field theory}

According to the standard model, the interaction of the matter particles is mediated by the gauge Bosons. The known electroweak, electromagnetic, and strong interactions have all turned out to be governed by the principles of the gauge invariance under local gauge transformations. The quantum field theories that have been proved useful in describing the real world are non-Abelian gauge theories. These are more general than the simple Abelian gauge invariance of the QED. 
In the standard model, the Lagrangian is invariant under a gauge transformation of the Fermion field $\hat{\psi}(x)$ of the material particle as follows:

$$
\delta \hat{\psi}(x)=i \theta^{\alpha}(x) t_{\alpha} \hat{\psi}(x),
$$

where $\theta^{\alpha}(x)$ is an arbitrary real function and where $t_{\beta}$ is the Hermitean operator which generates the unitary transformation. The $t_{\alpha}$ satisfies the Lie algebra:

$$
\begin{aligned}
& {\left[t_{\alpha}, t_{\beta}\right]=i C_{\gamma \alpha \beta} t_{\gamma},} \\
& C_{\gamma \alpha \beta} ; \text { completely antisymmetric, }
\end{aligned}
$$

where $C_{\gamma \alpha \beta}$ denotes the structure constant of the Lie algebra and is completely antisymmetric. The adjoint representation $t_{\alpha}^{A}$ defined as

$$
\left(t_{\alpha}^{A}\right)_{\beta \gamma}=-i C_{\beta \gamma \alpha}
$$

satisfies

$$
\left[t_{\alpha}^{A}, t_{\beta}^{A}\right]=i C_{\gamma \alpha \beta} t_{\gamma}^{A} .
$$

Since $\partial_{\mu} \hat{\psi}(x)$ does not transform like $\hat{\psi}(x)$, we must introduce a gauge potential $\hat{A}_{\alpha \mu}(x)$ and use it to construct the covariant derivative as follows:

$$
\hat{D}_{\mu}(x)=\partial_{\mu}+i \hat{A}_{\beta \mu}(x) t_{\beta}
$$

with the gauge transformation property

$$
\delta\left(\hat{D}_{\mu}(x) \hat{\psi}(x)\right)=i \theta^{\alpha}(x) t_{\alpha}\left(\hat{D}_{\mu}(x) \hat{\psi}(x)\right),
$$

if

$$
\begin{aligned}
& \delta \hat{A}_{\beta \mu}(x)=-\partial_{\mu} \theta^{\beta}(x)+\theta^{\alpha}(x) C_{\beta \gamma \alpha} \hat{A}_{\gamma \mu}(x) \\
& =-\hat{D}_{\mu}^{A} \theta^{\beta}(x)
\end{aligned}
$$

with

$$
\hat{D}_{\mu}^{A}(x)=\partial_{\mu}+i \hat{A}_{\beta \mu}(x) t_{\beta}^{A}
$$


The gauge potential $\hat{A}_{\alpha \mu}(x)$ leads to the gauge field density operator $\hat{F}_{\gamma \mu \nu}(x)$ as follows:

$$
\begin{aligned}
& {\left[\hat{D}_{\mu}(x), \hat{D}_{v}(x)\right]=i t_{\gamma} \hat{F}_{\gamma \mu \nu}(x),} \\
& \hat{F}_{\gamma \mu \nu}(x)=\partial_{\nu} \hat{A}_{\gamma \mu}(x)-\partial_{\mu} \hat{A}_{\gamma \nu}(x)-C_{\gamma \alpha \beta} \hat{A}_{\alpha \nu}(x) \hat{A}_{\beta \mu}(x),
\end{aligned}
$$

with the gauge transformation property

$$
\delta \hat{F}_{\gamma \mu \nu}(x)=i \theta^{\alpha}(x)\left(t_{\alpha}^{A}\right)_{\gamma \lambda} \hat{F}_{\lambda \mu \nu}(x) .
$$

The totally antisymmetric $C_{\gamma \alpha \beta}$ guarantees the following relationship

$$
\left[\hat{D}_{\mu}^{A}(x), \hat{D}_{\nu}^{A}(x)\right]=i t_{\gamma}^{A} \hat{F}_{\gamma \mu \nu}(x),
$$

and the Jacobi identity

$$
\hat{D}_{\lambda}^{A}(x) \hat{F}_{\alpha \mu \nu}(x)+\hat{D}_{\mu}^{A}(x) \hat{F}_{\alpha \nu \lambda}(x)+\hat{D}_{v}^{A}(x) \hat{F}_{\alpha \lambda \mu}(x)=0 .
$$

The last identity is an element of the Maxwell equations.

The gauge-invariant Lagrangian density operator $\hat{L}(x)$ with the non-Abelian gauge fields is generally written as

$$
\hat{L}(x)=-\frac{1}{16 \pi} \hat{F}_{\alpha \mu \nu}(x) \hat{F}_{\alpha}^{\mu v}(x)+\hat{L}_{M}\left(\left\{\hat{\psi}, \hat{D}_{\mu} \hat{\psi}\right\} ; x\right),
$$

where $\hat{L}_{M}\left(\left\{\hat{\psi}, \hat{D}_{\mu} \hat{\psi}\right\} ; x\right)$ is the Lagrangian density operator of the matter particle.

The equations of motion of fields is obtained using the variational principle.

First, the remaining elements of the Maxwell equations of motion are obtained for the non-Abelian fields in the gauge-invariant form using the Euler-Lagrange equation as follows:

$$
\hat{D}_{\mu}^{A}(x) \hat{F}_{\alpha}^{\mu \nu}(x)=\frac{4 \pi}{c} \hat{J}_{\alpha}^{v}(x),
$$

where $\hat{J}_{\alpha}^{v}(x)$ is the current of the matter field:

$$
\hat{J}_{\alpha}^{v}(x)=-c \frac{\delta \hat{L}_{M}\left(\left\{\hat{\psi}, \hat{D}_{\mu} \hat{\psi}\right\} ; x\right)}{\delta \hat{D}_{\mu}(x) \hat{\psi}(x)} i t_{\alpha} \hat{\psi}(x)
$$

The $\hat{J}_{\alpha}^{v}(x)$ satisfies the conservation law 


$$
\hat{D}_{\mu}^{A}(x) \hat{J}_{\alpha}^{\mu}(x)=0
$$

Second, let the matter spinor field Lagrangian density operator be given as follows:

$$
\hat{L}_{M}\left(\left\{\hat{\psi}, \hat{D}_{\mu} \hat{\psi}\right\} ; x\right)=c \hat{\bar{\psi}}(x)\left(i \hbar \gamma^{\mu} \hat{D}_{\mu}(x)-m c\right) \hat{\psi}(x),
$$

where $m$ denotes the mass of the matter particle. The matter field satisfies the equation of motion

$$
i \hbar \gamma^{\mu} \hat{D}_{\mu}(x) \hat{\psi}(x)=m c \hat{\psi}(x) .
$$

\section{A.2. The Lorentz force density and the spin torque density in the}

\section{non-Abelian gauge field theory}

We shall derive the stress tensor in non-Abelian gauge field theory and demonstrate its active role in the equation of motion of the kinetic momentum of the matter particle.

The kinetic momentum density operator $\hat{\vec{\Pi}}(x)$ of the matter particle defined as

$$
\begin{aligned}
& \hat{\vec{\Pi}}(x)=\frac{1}{2}\left(i \hbar \hat{\psi}^{\dagger}(x) \hat{\vec{D}}(x) \hat{\psi}(x)-i \hbar(\hat{\vec{D}}(x) \hat{\psi}(x))^{\dagger} \cdot \hat{\psi}(x)\right), \\
& \hat{\Pi}^{k}(x)=\frac{1}{2}\left(-i \hbar \hat{\psi}^{\dagger}(x) \hat{D}_{k}(x) \hat{\psi}(x)+i \hbar\left(\hat{D}_{k}(x) \hat{\psi}(x)\right)^{\dagger} \cdot \hat{\psi}(x)\right),
\end{aligned}
$$

satisfies the equation of motion

$$
\frac{\partial}{\partial t} \hat{\vec{\Pi}}(x)=\hat{\vec{L}}(x)+\hat{\vec{\tau}}^{\Pi}(x)
$$

where $\hat{\vec{L}}(x)$ denotes the material Lorentz force density operator and the $\hat{\vec{\tau}}^{\Pi}(x)$ denotes the material tension density operator. The $\hat{\vec{L}}(x)$ is given as follows:

$$
\hat{L}^{k}(x)=c \hbar \hat{F}_{\alpha \mu k}(x) \hat{\bar{\psi}}(x) \gamma^{\mu} t_{\alpha} \hat{\psi}(x) .
$$

The $\hat{\vec{\tau}}^{\Pi}(x)$ is given as the divergence of the material stress tensor density operator $\hat{\vec{\tau}}^{\Pi}(x)$

$$
\hat{\vec{\tau}}^{\Pi}(x)=\operatorname{div} \hat{\vec{\tau}}^{\Pi}(x),
$$




$$
\hat{\vec{\tau}}^{\Pi k}(x)=\partial_{l} \hat{\tau}^{\Pi k \ell}(x),
$$

where

$$
\hat{\tau}^{\Pi k \ell}(x)=\frac{i \hbar c}{2}\left[\hat{\bar{\psi}}(x) \gamma^{\ell} \hat{D}_{k}(x) \hat{\psi}(x)-\left(\hat{D}_{k}(x) \hat{\psi}(x)\right)^{\dagger} \gamma^{0} \gamma^{\ell} \cdot \hat{\psi}(x)\right]
$$

It should be noted that $\hat{\vec{\tau}}^{\Pi}(x)$ is Hermitean:

$$
\hat{\vec{\tau}}^{\Pi \dagger}(x)=\hat{\vec{\tau}}^{\Pi}(x) .
$$

Similar relationship holds in the Abelian gauge field theory: see Eqs. (2.37) and (2.43).

The Fermion spin angular momentum density operator $\frac{1}{2} \hbar \hat{\vec{\sigma}}(x)$ with

$$
\begin{aligned}
& \hat{\vec{\sigma}}(x)=\hat{\psi}^{\dagger}(x) \vec{\sigma} \hat{\psi}(x), \\
& \frac{1}{2} \hbar \sigma^{k}=\frac{1}{2} \varepsilon_{i j k} J^{i j}, \quad J^{i j}=\frac{1}{4} i \hbar\left[\gamma^{i}, \gamma^{j}\right],
\end{aligned}
$$

satisfies the equation of motion

$$
\frac{\partial}{\partial t}\left(\frac{1}{2} \hbar \hat{\vec{\sigma}}(x)\right)=\hat{\vec{t}}(x)+\hat{\vec{\zeta}}(x),
$$

where $\hat{\vec{t}}(x)$ denotes the spin-torque density operator defined as

$$
\hat{t}^{k}(x)=-\varepsilon_{i j k} \hat{\tau}^{\Pi i j}(x) .
$$

The $\hat{\vec{\zeta}}(x)$ denotes the zeta force density operator defined as

$$
\hat{\zeta}^{k}(x)=-c \partial_{k}\left(\hat{\bar{\psi}}(x) \gamma^{k} \frac{1}{2} \hbar \sigma^{k} \hat{\psi}(x)\right) ; \text { no sum over } \mathrm{k} .
$$

The alternative form is obtained as follows:

$$
\begin{aligned}
& \hat{\zeta}^{k}(x)=-\partial_{k} \hat{\phi}_{5}(x), \\
& \hat{\phi}_{5}(x)=\frac{\hbar}{2 q} \hat{j}_{5}^{0}(x),
\end{aligned}
$$

where $q$ denotes the charge $q=Z e$ of the matter particle and $\hat{j}_{5}^{0}(x)$ denotes the zeroth component of the chiral current density operator 


$$
\begin{aligned}
& \hat{j}_{5}^{\mu}(x)=c q \hat{\bar{\psi}}(x) \gamma^{\mu} \gamma_{5} \hat{\psi}(x), \\
& \gamma_{5}=i \gamma^{0} \gamma^{1} \gamma^{2} \gamma^{3} .
\end{aligned}
$$

Similar relationship holds in Abelian gauge field theory: see Eqs. (4. 3) - (4.10).

\section{A.3. Spontaneously broken symmetry}

Using the standard model, the gauge fields of QCD is reduced from the grand unified theory $(\mathrm{GUT})$ as $S U(3)_{\mathrm{c}} \times S U(2)_{\mathrm{w}} \times U(1)_{\mathrm{y}} \rightarrow S U(3)_{\mathrm{c}} \times U(1)_{\mathrm{QED}}$, where the Higgs field breaks the Weinberg-Salam electroweak gauge group $S U(2)_{\mathrm{w}} \times U(1)_{\mathrm{y}}$ down to $U(1)_{\mathrm{QED}}$, but the color and charge symmetries remain intact.

The Hamiltonian density operator in the field theory is obtained by canonical quantization using Lagrangian density operator:

$$
\begin{aligned}
& \hat{L}(x)=\hat{L}_{\text {Gauge fields }}(x)+\hat{L}_{\text {Material particle fields }}(x) \\
& +\hat{L}_{\text {Higgs field }}(x)+\hat{L}_{\text {Yukawa coupling }}(x)
\end{aligned}
$$

The matter particles are spin 1/2 chiral Fermions in standard model, accompanying the three gauge symmetries.

First, quarks has internal color symmetry of the $S U(3)_{\text {c }}$ gauge:

$$
\hat{\psi}_{f}=\left(\begin{array}{c}
\hat{q}_{\text {red }} \\
\hat{q}_{\text {blue }} \\
\hat{q}_{\text {green }}
\end{array}\right)_{f},
$$

with the gauge transformation property

$$
\hat{\psi}_{f}^{\left(\theta_{c}\right)}=\exp \left(i \theta_{c}^{\ell}(x) \frac{g_{c}}{\hbar c} \frac{\lambda_{\ell}}{2}\right) \hat{\psi}_{f},
$$

and the covariant derivative

$$
\hat{D}_{\mu}(x)=\partial_{\mu}+i \frac{g_{c}}{\hbar c} \frac{\lambda_{\ell}}{2} \hat{G}_{\mu}^{\ell}(x) .
$$

Flavour $f$ are

$$
u_{R, L}, d_{R, L}, c_{R, L}, s_{R, L}, t_{R, L}, b_{R, L} \text {, }
$$


where $R, L$ denotes chirality

$$
\frac{1}{2}\left(1+\gamma_{5}\right) \hat{\psi}(x)=\hat{\psi}_{R}(x), \quad \frac{1}{2}\left(1-\gamma_{5}\right) \hat{\psi}(x)=\hat{\psi}_{L}(x) .
$$

Second, $S U(2)_{\mathrm{w}}$ gauge of weak isospin doublets (Cabibbo-rotated quark $d_{c L}$ ):

$$
\begin{aligned}
& \left(\begin{array}{l}
\psi_{u_{L}}{ }^{\left(\theta_{t}\right)}(x) \\
\psi_{d_{c L}}{ }^{\left(\theta_{t}\right)}(x)
\end{array}\right)=\exp \left(i \theta_{t}^{k}(x) \frac{g_{t}}{\hbar c} t_{k}\right)\left(\begin{array}{l}
\psi_{u_{L}}(x) \\
\psi_{d_{c L}}(x)
\end{array}\right): t_{3}=\frac{1}{2},-\frac{1}{2} \\
& \left(\begin{array}{l}
\psi_{v_{e}}{ }^{\left(\theta_{t}\right)}(x) \\
\psi_{e_{L}}{ }^{\left(\theta_{t}\right)}(x)
\end{array}\right)=\exp \left(i \theta_{t}^{k}(x) \frac{g_{t}}{\hbar c} t_{k}\right)\left(\begin{array}{l}
\psi_{v_{e}}(x) \\
\psi_{e_{L}}(x)
\end{array}\right): t_{3}=\frac{1}{2},-\frac{1}{2}
\end{aligned}
$$

and singlets

$$
u_{R}, d_{c R}, e_{R}: t=0 .
$$

The quarks are mixed through the first and second generations by the Cabibbo angle $\theta_{\mathrm{c}}$

$$
\left(\begin{array}{l}
\psi_{d_{c}}(x) \\
\psi_{s_{c}}(x)
\end{array}\right)=\left(\begin{array}{cc}
\cos \theta_{c} & \sin \theta_{c} \\
-\sin \theta_{c} & \cos \theta_{c}
\end{array}\right)\left(\begin{array}{l}
\psi_{d}(x) \\
\psi_{s}(x)
\end{array}\right),
$$

or more generally through the first, second, and third generations by the Cabibbo-Kobayashi-Maskawa matrix

$$
\left(\begin{array}{l}
\psi_{d^{\prime}}(x) \\
\psi_{s^{\prime}}(x) \\
\psi_{b^{\prime}}(x)
\end{array}\right)=\left(\begin{array}{lll}
V_{u d} & V_{u s} & V_{u b} \\
V_{c d} & V_{c s} & V_{c b} \\
V_{t d} & V_{t s} & V_{t b}
\end{array}\right)\left(\begin{array}{l}
\psi_{d}(x) \\
\psi_{s}(x) \\
\psi_{b}(x)
\end{array}\right) .
$$

Third, $U(1)_{y}$ gauge of weak hypercharge:

$$
\begin{aligned}
& \psi_{u_{R, L}}\left(\theta_{y}\right)(x)=\exp \left(i \theta_{y}(x) \frac{g_{y}}{\hbar c} \frac{y_{u_{R, L}}}{2}\right) \psi_{u_{R, L}}(x): y_{u_{R}}=\frac{4}{3}, y_{u_{L}}=\frac{1}{3} \\
& \psi_{d_{c R, L}}{ }^{\left(\theta_{y}\right)}(x)=\exp \left(i \theta_{y}(x) \frac{g_{y}}{\hbar c} \frac{y_{d_{R, L}}}{2}\right) \psi_{d_{c R, L}}(x): y_{d_{c R}}=-\frac{2}{3}, y_{d_{c L}}=\frac{1}{3} \\
& \psi_{v_{e}}^{\left(\theta_{y}\right)}(x)=\exp \left(i \theta_{y}(x) \frac{g_{y}}{\hbar c} \frac{y_{v_{e}}}{2}\right) \psi_{v_{\mathrm{e}}}(x):---, y_{v_{\mathrm{e}}}=-1 \\
& \psi_{e_{R, L}}{ }^{\left(\theta_{y}\right)}(x)=\exp \left(i \theta_{y}(x) \frac{g_{y}}{\hbar c} \frac{y_{e_{R, L}}}{2}\right) \psi_{e_{R, L}}(x): y_{e_{R}}=-2, y_{e_{L}}=-1
\end{aligned}
$$

for the $1^{\text {st }}$ generation $u, d, v_{e}, e$ and similar relationships for the $2^{\text {nd }}$ generation $c, s, v_{\mu}, \mu$ and $3^{\text {rd }}$ generation $t, b, v_{\tau}, \tau$.

The charge $q=Z e$ of $S U(2)_{\mathrm{w}} \times U(1)_{\mathrm{y}}$ are found to be 


$$
\begin{aligned}
& Z=t_{3}+\frac{y}{2} \\
& Z_{u_{R, L}}=\frac{2}{3}, Z_{d_{c R, L}}=-\frac{1}{3}, Z_{v_{e}}=0, Z_{e_{R, L}}=-1
\end{aligned}
$$

for the $1^{\text {st }}$ generation $u, d, v_{e}, e$ and similar relationships for the $2^{\text {nd }}$ generation $c, s, v_{\mu}, \mu$ and $3^{\text {rd }}$ generation $t, b, v_{\tau}, \tau$.

The matter particles are bound by gauge bosons obeying gauge principle represented by the covariant derivative operator as

$$
\hat{D}_{\mu}(x)=\partial_{\mu}+i \frac{g_{c}}{\hbar c} \frac{\lambda_{\ell}}{2} \hat{G}_{\mu}^{\ell}(x)+i \frac{g_{t}}{\hbar c} t_{k} \hat{W}_{\mu}^{k}(x)+i \frac{g_{y}}{\hbar c} \frac{y}{2} \hat{X}_{\mu}(x) .
$$

Quarks are bound by gluons $G_{\mu}^{\ell}$ while electron acquires its charge and mass through Higgs mechanism with Yukawa coupling, when massless photon $A_{\mu}$ as well as massive bosons $Z_{\mu}^{0}$ and $W_{\mu}^{ \pm}$are emerging using Glashow-Weinberg-Salam theory of spontaneously broken gauge symmetry as follows:

$$
\begin{aligned}
& i \frac{g_{t}}{\hbar c} t_{k} \hat{W}_{\mu}^{k}(x)+i \frac{g_{y}}{\hbar c} \frac{y}{2} \hat{X}_{\mu}(x)=i \frac{g_{t}}{\hbar c} \sin \theta_{W} Z \hat{A}_{\mu}(x) \\
& +i \frac{g_{t}}{\hbar c} \frac{1}{\cos \theta_{W}}\left(t_{3}-\sin ^{2} \theta_{W} Z\right) \hat{Z}_{\mu}^{0}(x) \\
& +i \frac{g_{t}}{\hbar c} \frac{1}{\sqrt{2}}\left(t_{-} \hat{W}_{\mu}^{+}(x)+t_{+} \hat{W}_{\mu}^{-}(x)\right)
\end{aligned}
$$

where

$$
\begin{aligned}
& \hat{A}_{\mu}(x)=\cos \theta_{W} \hat{X}_{\mu}(x)+\sin \theta_{W} \hat{W}_{\mu}^{3}(x) \\
& \hat{Z}_{\mu}^{0}(x)=-\sin \theta_{W} \hat{X}_{\mu}(x)+\cos \theta_{W} \hat{W}_{\mu}^{3}(x) \\
& \hat{W}_{\mu}^{ \pm}(x)=\frac{1}{\sqrt{2}}\left(\hat{W}_{\mu}^{1}(x) \pm i \hat{W}_{\mu}^{2}(x)\right) \\
& \cos \theta_{W}=\frac{g_{t}}{\sqrt{g_{t}^{2}+g_{y}^{2}}}, \sin \theta_{W}=\frac{g_{y}}{\sqrt{g_{t}^{2}+g_{y}^{2}}} \\
& t_{ \pm}=t_{1} \pm i t_{2}
\end{aligned}
$$

Then, the charge of electron emerges as

$$
e=g_{t} \sin \theta_{W} ; \quad \text { charge of electron }(\times(-1)) \text {. }
$$


The Higgs field $\hat{H}(x)$ itself is an isospinor scalar field with weak hypercharge $y=+1 / 2$ :

$$
\hat{H}(x)=\left(\begin{array}{c}
\hat{H}^{+}(x) \\
\hat{H}^{0}(x)
\end{array}\right),
$$

spontaneously broken if the potential $\hat{V}(\hat{H}(x))$ has the minimum at $v$ as

$$
\hat{H}(x)=\left(\begin{array}{c}
0 \\
v+\hat{\sigma}(x)
\end{array}\right),
$$

with real $v$ and $\hat{\sigma}(x)$.

As a result, the Yukawa coupling Lagrangian density $\hat{L}_{\text {Yukawa coupling }}(x)$ turned out to be

$$
\begin{aligned}
& \hat{L}_{\text {Yukawa coupling }}(x)=-G_{e}\left(\hat{\bar{\psi}}_{e_{R}}(x) \hat{H}^{\dagger}(x) \hat{\psi}_{e_{L}}(x)+\hat{\bar{\psi}}_{e_{L}}(x) \hat{H}(x) \hat{\psi}_{e_{R}}(x)\right) c^{2} . \\
& =-G_{e}(v+\hat{\sigma}(x)) c^{2} \hat{\bar{\psi}}_{e}(x) \hat{\psi}_{e}(x)
\end{aligned}
$$

It follows that the mass of electron emerges as

$m_{e}=G_{e} v ;$ mass of electron .

Moreover, the kinetic term of the Higgs field Lagrangian density $\hat{L}_{\text {Higgs field }}(x)$ with real $v$ and $\hat{\sigma}(x)$ turned out to be:

$$
\begin{aligned}
& \hat{L}_{\text {Higgs field }}(x)=\frac{\hbar^{2}}{2}\left(\hat{D}^{\mu}(x) \hat{H}(x)\right)^{\dagger}\left(\hat{D}_{\mu}(x) \hat{H}(x)\right)-\hat{V}(\hat{H}(x)) \\
& =\frac{\hbar^{2}}{2}\left(\partial^{\mu} \hat{\sigma}(x)\right)\left(\partial_{\mu} \hat{\sigma}(x)\right) \\
& +\frac{\hbar^{2}}{2} \frac{1}{4}\left(\frac{g_{T}}{\hbar c}\right)^{2}\left(\left(\hat{W}^{1}(x)\right)^{2}+\left(\hat{W}^{2}(x)\right)^{2}\right)(v+\hat{\sigma}(x))^{2} \\
& +\frac{\hbar^{2}}{2} \frac{1}{4}\left(\frac{g_{T}}{\hbar c}\right)^{2} \frac{1}{\cos ^{2} \theta_{W}}\left(\hat{Z}^{0}(x)\right)^{2}(v+\hat{\sigma}(x))^{2} \\
& -\hat{V}(\hat{H}(x))
\end{aligned}
$$

This demonstrates that the massive bosons $Z_{\mu}{ }^{0}$ and $W_{\mu}{ }^{ \pm}$are emerging while the massless photon $A_{\mu}$ remained intact. 


\section{References}

[1] P. Hohenberg and W. Kohn, Phys. Rev. 136, B864 (1964).

[2] N. D. Mermin, Phys. Rev. A 137, 1441 (1965).

[3] R. G. Parr and W. Yang, Density Functional Theory of Atoms and Molecules (Oxford Univ., 1989).

[4] A. Tachibana, In Fundamental Perspectives in Quantum Chemistry: A Tribute to the Memory of Per-Olov Löwdin, Edited by E. Brändas and E. Kryachko (Dordrecht, Kluwer, 2003) Volume 2, pp. 211-39.

[5] A .Tachibana, Int. J. Quant. Chem. 100, 981 (2004).

[6] A. Tachibana, In Reviews in Modern Quantum Chemistry: A Celebration of the Contributions of Robert Parr, Edited by K. D. Sen (Singapore, World Scientific, 2002), Volume 2, Chapter 45, pp. 1327-66.

[7] A. Tachibana, J. Mol. Model. 11, 301 (2005).

[8] A. Tachibana, J. Chem. Phys. 115, 3497 (2001).

[9] A. Tachibana, In Stress Induced Phenomena in Metallization, Edited by S. P. Baker (New York, American Institute of Physics, 2002), pp 105-16.

[10] P. Szarek and A. Tachibana, J. Mol. Model. 13, 651 (2007).

[11] P. Szarek, Y. Sueda, and A. Tachibana, J. Chem. Phys. 129, 094102 (2008).

[12] P. Szarek, K. Urakami, C. Zhou, H. Cheng, and A. Tachibana, J. Chem. Phys. 130, 084111 (2009).

[13] W. Pauli, Handbuch der Physik, Band XXIV, Teil 1 (Springer, Berlin, 1933), pp. 83-272; reprinted In Handbuch der Physik (Springer, Berlin, 1958), Vol. 5, Part1; translated into English In General Principles of Quantum Mechanics (Berlin, Springer, 1980).

[14] E. Schödinger, Ann. Phys. (Leipzig) 82, 265 (1927).

[15] R. F. W. Bader, Atoms in Molecules: A Quantum Theory (Oxford, Clarendon, 1990).

[16] Saul T. Epstein, J. Chem. Phys. 63, 3573 (1975).

[17] A. Tachibana, Int. J. Quantum Chem., 35, 361 (1989).

[18] A. Tachibana, Theoretical Chemistry Accounts, 102, 188 (1999).

[19] A. Tachibana and R. G. Parr, Int. J. Quant. Chem., 41, 527 (1992).

[20] C. P. Slichter, Principles of Magnetic Resonance (Berlin, Springer-Verlag, 1990), Chapter 4.

[21] R. Krause-Rehberg and H. S. Leipner, Positron Annihilation in Semiconductors, Solid State Sciences, Volume 127 (Berlin, Springer-Verlag, 1999).

[22] S. Weinberg, The Quantum Theory of Fields (Cambridge, Cambridge Univ., 1995).

[23] J. D. Jackson, Classical Electrodynamics (New York, John Wiley, 1998), Chapter 11.

[24] A. Lodder and J. P. Dekker, In Stress Induced Phenomena in Metallization, Edited by Okabayashi et al . ( New York, American Institute of Physics, 1998) , pp 315-28. 
[25] C. Bosvieux and J. Friedel, J. Phys. Chem. Solids 23, 123 (1962).

[26] N. W. Ashcroft and N. D. Mermin, Solid State Physics (New York, Thomson Learning, 1976), Chapter 1.

[27] A. C. Dillon, K. M. Jones, T. A. Bekkedahl, C. H. Kiang, D. S. Bethune, and M. J. Heben, Nature 386, 377 (1997).

[28] P. Chen, X. Wu, J. Lin, and K. L. Tan, Science 285, 91 (1999).

[29] L. Jeloacia and V. Sidis, Chem. Phys. Lett. 300, 157 (1999).

[30] V. Sidis, L. Jeloaica, A. G. Borisov, and S. A. Deutscher, In Molecular Hydrogen in Space, Edited by F. Combes and G. Pineau des Forê ts (Cambridge University, New York, 2000), pp. 89-97. [31] A. Zuttel, P. Sudan, Ph. Mauron, T. Kiyobayashi, Ch. Emmernegger, and L. Schlapbach, Int. J. Hydrogen Energy 27, 203 (2002).

[32] F. L. Darkrim, P. Malbrunot, and G. P. Tartaglia, Int. J. Hydrogen Energy 27, 193 (2002).

[33] G. E. Froudakis, J. Phys.: Cond. Matter 14, R453 (2002).

[34] E.-C. Lee, Y.-S. Kim, Y.-G. Jin, and K. J. Chang, Phys. Rev. B 66, 073415 (2002).

[35] X. Sha and B. Jackson, Surf. Sci. 496, 318 (2002).

[36] Y. Miura, H. Kasai, W. A. Di ñ o, H. Nakanishi, and T. Sugimoto, Jpn. J. Appl. Phys. 42, 4626 (2003); ibid., 72, 995 (2003); ibid., 93, 3395 (2003).

[37] H. Cheng, A. C. Cooper, and G. P. Pez, J. Am. Chem. Soc. 123, 5845 (2001);

L. Chen, A. C. Cooper, G. P. Pez, and H. Cheng, J. Phys. Chem. C, in press.

[38] A. Chambers, C. Park, R. T. K. Baker, J. Phys. Chem. B 102, 4253 (1998);

Y. Ye et al., Appl. Phys. Lett. 74, 2307 (1999).

[39] M. Rutigliano, M. Cacciatore, and G. D. Billing, Chem. Phys. Lett. 340, 13 (2001).

[40] R. T. Yang, Carbon 38, 623 (2000).

[41] F. Pinkerton, B. Wickle, C. Olk, G. Tibbetts, M. S. Meyer, and J. Herbst, Proceedings of the 10th Canadian Hydrogen Energy Conference, Beiging, China, Published by International Hydrogen Association, 560 (2000).

[42] H. Cheng, G. Pez, G. Kern, G. Kresse, and J. Hafner, J. Phys. Chem. B 105, 736 (2001).

[43] H. Nakano, H. Ohta, K. Kawano, K. Doi, and A. Tachibana, unpublished.

[44] M. Senami, K. Ichikawa, P. Szarek, K. Doi, K. Nakamura, and A. Tachibana, Molecular Regional DFT program package, ver. 3, Tachibana Lab., Kyoto Univ., 2008. 